\title{
A Semianalytical Approach for Nonlinear Dynamic System of Shallow Arches Using Higher Order Multistep Taylor Method
}

\author{
Sudeok Shon $\mathbb{D}^{1},{ }^{1}$ Soohong Ahn, ${ }^{2}$ Seungjae Lee $\mathbb{D}^{\mathbb{D}},{ }^{3}$ and Junhong $\mathrm{Ha}^{4}$ \\ ${ }^{1}$ Department of Architectural Engineering, Korea University of Technology and Education, Cheonan 31253, Republic of Korea \\ ${ }^{2}$ Acrovision Co. Ltd., B-1411, 70 Dusan-ro, Geumcheon-gu, Seoul 08584, Republic of Korea \\ ${ }^{3}$ Interdisciplinary Program in Creative Engineering, Korea University of Technology and Education, Cheonan 31253, Republic of Korea \\ ${ }^{4}$ School of Liberal Arts, Korea University of Technology and Education, Cheonan 31252, Republic of Korea
}

Correspondence should be addressed to Seungjae Lee; leeseung@koreatech.ac.kr

Received 21 November 2017; Revised 21 April 2018; Accepted 24 April 2018; Published 19 August 2018

Academic Editor: Stefano Lenci

Copyright (c) 2018 Sudeok Shon et al. This is an open access article distributed under the Creative Commons Attribution License, which permits unrestricted use, distribution, and reproduction in any medium, provided the original work is properly cited.

\begin{abstract}
This study aimed at obtaining a semianalytical solution for nonlinear dynamic system of shallow arches. Taylor method was applied to find the analytical solution, and an investigation of their dynamic characteristic was carried out to verify the applicability of this methodology for the shallow arches under step or periodic excitation. A polynomial solution can be obtained from this multistep approach with respect to time, and direct buckling as well as indirect buckling of the shallow arches can be observed, also. The results indicated that the dynamic buckling load level was higher with higher shape factor. Additionally, a change of attractor in phase space was investigated. Coupling in symmetric mode as well as asymmetric mode was observed in case of indirect buckling, and a sensitive response was also manifested during sinusoidal and beating excitation. These results of applying multistep Taylor series for the investigation of displacement response and attractor change revealed that this analytical approach was valid in explaining the dynamic buckling behavior of shallow arches under direct and indirect snapping.
\end{abstract}

\section{Introduction}

Large span roof structures are generally designed as arches and spherical shells. In other words, the structural performance of the arch depends on its shape. Thus, lighter, thinner, and larger space can be made with less materials compared to traditional flat structures. Shallow arches have received considerable attention in the field of architecture because of their beautiful shape and economic efficiency. However, the response to transverse loading of the shallow arch is quite different from that of the flat beam. The structural design also requires dynamic behavior analysis under various loads. Since a large roof can be treated separately from the substructures, the arch's boundary is usually designed by hinge, and with shallow arch roofs the primary interest is in the instability of vertical direction behavior. The dynamic unstable behavior of shallow arches is generally complex depending on the initial condition, e.g., geometrically imperfection, and shape of the arches. In particular, direct snapping in symmetric mode and indirect snapping due to coupling in asymmetric mode manifest very sensitive behavior and progress to chaotic behavior, attracting deep interest from many researchers.

Investigation of dynamic instability of shallow arches has started with the fundamental study of Hoff and Bruce [1] in the mid-1950s and was followed by many researches afterwards in the beginning (Humphreys [2]; Lock [3]; Hsu [4]; Ariaratnam and Sankar [5]; Sundararajan and Kumani [6]; Donaldson and Plaut [7]; and Kounadis et al. [8]). These early researches on shallow arches resulted in rebounding studies of chaotic motion or global dynamic behavior (Blair et al. [9]; Levitas et al. [10]), accurate solution of free-vibration (Tseng et al. [11]; De Rosa and Franciosi [12]), internal resonance (Bi and Dai [13]; Lacarbonara and Rega [14]), and buckling under boundary condition and moving load (Kong et al. [15]; Chen and Lin [16, 17]). Recently, many investigations for design and construction of shallow arch roofs have been conducted, including the identification and stability for shallow arches (Ha et al. [18]), and sensitivity of shallow arch (Virgin et al. [19]). On the other hand, the initially 
curved or buckled microbeams in mechanics (Farokhi and Ghayesh [20]) have been studied in terms of nonlinearity, geometrical imperfection, resonance, and chaotic behaviors. Various numerical researches have been conducted on the nonlinear behaviors of the microbeams and their application. However, this buckled configuration of the microbeams is due to some external effects such as temperature increase or compressive forces, which are not associated with the arch roof. Even though in the study of arch roofs the range of arch shapes and sizes differs from that of microbeams, these investigations are still useful for all engineering fields. The evaluation of dynamic buckling of shallow arch roof generally uses energy method or phase space of system approach or direct approach (Lin and Chen [21]) of investigating significant growth of displacement response (Budiansky and Roth [22]) by obtaining dynamic response of the governing equations in a wide range of parameters. This process of dealing with dynamic loading requires numerical method (Belytschko [23]; Argyris et al. [24]) or analytical techniques for solving nonlinear differential equations, and the process of formulating the equations differs by the methodology. One of the direct purposes of dynamic analysis is finding out the transient response to external force and ultimately attaining the solution to motion equations. However, it is difficult to obtain an analytical solution for dynamic system of shallow arches under the various external forces.

The techniques to obtain an analytical solution to nonlinear differential equations have been considerably investigated in the past (Sadighi et al. [25]). Analytical or semianalytical methods include the traditional Taylor's power series method (Barrio [26]; Barrio et al. [27]), Adomian decomposition method (Adomian and Rach [28]; Adomian and Rach [29]), homotopy analysis method (Liao [30]), and homotopy perturbation method (He [31]; He [32]; and Chowdhury et al. [33]). Unlike numerical ones, these methods are limited in their applicability and are heavily dependent on the influence of their parameters. The Taylor series method, which has the longest history, provides an accurate analytical solution with excellent stability. Recently, Barrio et al. [27] explained that the Taylor series method provides advantages including easy formation with the method of using variable order and step-size and usefulness in many dynamic systems requiring a high-precision solution. In particular, when we need to perform long-time numerical simulations and sometimes to look for high precision solutions of differential systems, the family of ordinary differential equation solvers that can answer the requirements is the Taylor Series Method (Barrio et al. [34]). Additionally, it has been reported that appropriately adjusting the number of terms and error limit is advantageous in computing the error limit and attaining an accurate solution fairly easily (Barrio et al. [27]; Shon et al. [35]). Traditional Taylor series method requires an infinite series expansion for attaining periodic response of a dynamic system and can be inappropriate for discrete nonlinear governing equations. However, the Taylor series method expanding in multistep can compute a high precision analytical solution with relatively less degree of differential coefficients. Therefore, this study applied multistep Taylor series method to compute an accurate semianalytical solution

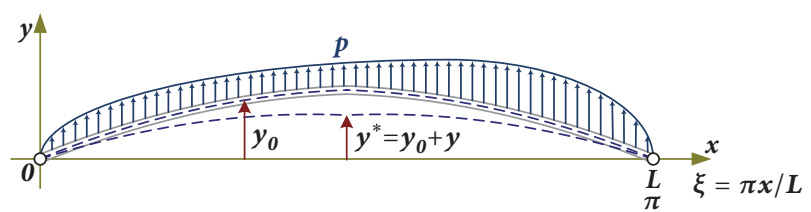

FIgURE 1: The shape of a pin-ended shallow sinusoidal arch under distributed dynamic loading.

of a dynamic governing equation of motion for shallow sinusoidal arches and examined their dynamic response and attractor of phase space to determine its applicability. This paper is organized as follows. Sections 2 and 3 describe the formulation of nonlinear governing equation of motion for shallow arches and theoretical analysis technique of multistep Taylor series method, respectively. Section 4 computes a multistep Taylor series solution for the nonlinear governing equation of motion under symmetric mode and investigates the dynamic behavior of shallow arches. Section 5 examines the dynamic response and attractor in phase space of the system for an indirect snapping model under asymmetric mode and periodic excitation. Last, Section 6 concludes this paper.

\section{Nonlinear Equations of Motion}

The governing equation of motion is expressed in the following equation when the shape and coordinate system of the shallow arches are defined as in Figure 1 (Lin and Chen [21]; Ha et al. [18]). In this figure, the two ends of the arch are separated from each other by the distance $L$. The original shape of the unloaded arch is denoted by $y_{0}(x)$, and the vertical and longitudinal deflections are expressed as a function of $y(x, t)$ and $u(x, t)$, respectively. The arch system is subjected to vertical loading $p(x, t)$ within the $(x, y)$ coordinate system, and a dimensionless parameter, $\xi=\pi x / L$, is used. Besides, the constant parameters of the system are density of mass $\rho$, Young's modulus $E$, cross-sectional area $A(x)$, and moment of inertia $I(x)$.

The kinetic energy of the shallow arches can be expressed as follows:

$$
T=\frac{\rho A}{2} \int_{0}^{L}\left(\frac{\partial y}{\partial t}\right)^{2} d x
$$

The potential energy (strain energy) $U$ of the system consists of two parts, as shown in (2), one $\left(U_{a}\right)$ due to the axial force and one $\left(U_{b}\right)$ due to bending. The energy can be expressed as follows:

$$
U=U_{a}+U_{b}=\frac{E A}{2} \int_{0}^{L} \varepsilon_{0}^{2} d x+\frac{E I}{2} \int_{0}^{L} \kappa^{2} d x,
$$

where axial strain $\varepsilon_{0}$ and curvature $\kappa$ of the shallow arches using Green's strain tensor are as follows:

$$
\begin{aligned}
\varepsilon_{0} & =\frac{\partial u}{\partial x}+\frac{\partial y_{0}}{\partial x} \frac{\partial y}{\partial x}+\frac{1}{2}\left(\frac{\partial y}{\partial x}\right)^{2} \\
\kappa & =\frac{\partial^{2} y}{\partial x^{2}}
\end{aligned}
$$


There are two types of external forces exerting on the arch: excitation force $p(x, t)$ and the damping force. Dissipative force, damping, is considered a uniformly distributed viscous damping force only in the transversal direction. As such, the external works by the external force and dissipative force are as follows:

$$
W_{n c}=W_{d}-W_{e x t}=-\int_{0}^{L} c \frac{\partial y}{\partial t} y d x+\int_{0}^{L} p y d x
$$

where $c$ is the viscous damping coefficient. Now, consider that axial force $N$ is constant $\left(=E A \varepsilon_{0}\right)$, and $\partial N / \partial x=0$ and the boundary condition at $x=0, L$ is $u(0)=u(L)=0$. Then, the $N(x)$ can be expressed as follows:

$$
N=\frac{E A}{2 L} \int_{0}^{L}\left(2 \frac{\partial y_{0}}{\partial x} \frac{\partial y}{\partial x}+\left(\frac{\partial y}{\partial x}\right)^{2}\right) d x
$$

The variations of potential energy $U$, kinetic energy $T$, and work $W_{n c}$ can be formulated as follows:

$$
\begin{gathered}
\delta T=-\rho A \int_{0}^{L} \frac{\partial^{2} y}{\partial t^{2}} \delta y d x \\
\delta\left(U_{a}+U_{b}\right)=\int_{0}^{L} N\left(\frac{\partial^{2} y_{0}}{\partial x^{2}}+\frac{\partial^{2} y}{\partial x^{2}}\right) \delta y d x \\
-\int_{0}^{L} E I \frac{\partial^{4} y}{\partial x^{4}} \delta y d x \\
\delta W_{n c}=-\int_{0}^{L} c \frac{\partial y}{\partial t} \delta y d x+\int_{0}^{L} p \delta y d x
\end{gathered}
$$

Equations (7)-(9) are substituted to extended Hamilton's principle given by

$$
\int_{t_{1}}^{t_{2}} \delta T-\delta\left(U_{a}+U_{b}\right)+\delta W_{n c} d t=0,
$$

which yields the following nonlinear partial differential equations governing the motion of the shallow arch system (Ha et al. [18]):

$$
\begin{array}{r}
E I \frac{\partial^{4} y}{\partial x^{4}}-\left[\frac{E A}{2 L} \int_{0}^{L}\left\{\left(\frac{\partial y}{\partial x}\right)^{2}+2 \frac{\partial y_{0}}{\partial x} \frac{\partial y}{\partial x}\right\} d x\right] \\
\cdot\left(\frac{\partial^{2} y_{0}}{\partial x^{2}}+\frac{\partial^{2} y}{\partial x^{2}}\right)+c \frac{\partial y}{\partial t}+\rho A \frac{\partial^{2} y}{\partial t^{2}}-p=0
\end{array}
$$

The following parameters are used to express the above equation (11) with dimensionless variables. Here, $k$ represents the radius of gyration of the cross-section.

$$
\begin{aligned}
\eta_{0} & =\frac{y_{0}}{k}, \\
\eta & =\frac{y}{k}, \\
q & =\frac{p}{E I k}\left(\frac{L}{\pi}\right)^{4},
\end{aligned}
$$

$$
\begin{aligned}
\tau & =w_{0} t \\
w_{0} & =\left(\frac{\pi}{L}\right)^{2}\left(\frac{E I}{\rho A}\right)^{1 / 2}, \\
\gamma & =\frac{c}{\rho A w_{0}}
\end{aligned}
$$

Thus, (11) above can be expressed as the following by using dimensionless system $\xi$ of Figure 1 .

$$
\begin{aligned}
& \frac{\partial^{4} \eta}{\partial \xi^{4}} \\
& -\left[\frac{1}{2 \pi} \int_{0}^{\pi}\left\{\left(\frac{\partial \eta}{\partial \xi}\right)^{2}+2 \frac{\partial \eta_{0}}{\partial \xi} \frac{\partial \eta}{\partial \xi}\right\} d \xi\right]\left(\frac{\partial^{2} \eta_{0}}{\partial \xi^{2}}+\frac{\partial^{2} \eta}{\partial \xi^{2}}\right) \\
& +\gamma \frac{\partial \eta}{\partial t}+\frac{\partial^{2} \eta}{\partial t^{2}}-q=0
\end{aligned}
$$

The initial shape, $\eta_{0}$, deflection $\eta$, and transverse loading $q$ of sinusoidal arches are defined as the following. The $\eta$ representing assumed deformed shape of the arch satisfies the boundary condition $\left(y(0)=y(L)=0 ; y^{\prime \prime}(0)=y^{\prime \prime}(L)=0\right)$ of a pin-ended arch.

$$
\begin{aligned}
\eta_{0} & =h \sin \xi \\
\eta & =\sum_{i=1}^{N} D_{i} \sin (i \xi), \\
q & =\Lambda \sin \xi
\end{aligned}
$$

Accordingly, substitution of the assumed shape, deflection, and load to equation (13) results in nonlinear governing equations of motion for the coefficients $D_{n}$ of the dimensionless deflection function.

$$
\begin{aligned}
\ddot{D}_{j}= & \Lambda \delta_{1 j}-\gamma \dot{D}_{j}-j^{4} D_{j} \\
& -\frac{1}{4}\left(\sum_{i=1}^{N} i^{2} D_{i}{ }^{2}-2 h D_{1}\right)\left(-h \delta_{1 j}+j^{2} D_{j}{ }^{2}\right), \\
& \quad j=1,2, \ldots,
\end{aligned}
$$

where $\delta_{i j}$ is Kronecker delta, and solving the above equation can explain the dynamic behavior of the arch. Additionally, since $D_{1}$ and $D_{2}$ are amplitudes of symmetric mode and asymmetric mode, respectively, the indirect snapping phenomenon can be explained by considering the $D_{2}$ term.

\section{Taylor Series Using Order and Step Parameter}

This chapter discusses the formulation of Taylor series analytical method and its governing equations. Equation (15) is defined so as to explain the Taylor series method for computing the solution to initial value problem of nonlinear 


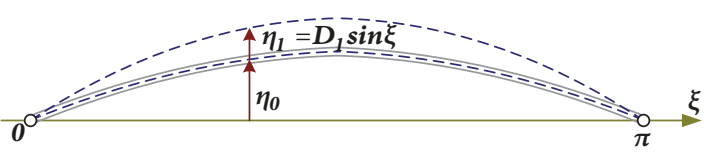

(a)

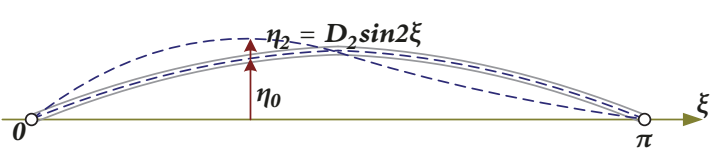

(b)

Figure 2: Deflection modes of the shallow arches: (a) symmetric mode (1st mode) and (b) asymmetric mode (2nd mode).

governing equations and its approximate Taylor series solution.

$$
\begin{aligned}
\dot{\ddot{D}} & =f(t, \dot{\boldsymbol{D}}, \boldsymbol{D}), \quad t \in I, \boldsymbol{D}=\boldsymbol{D}(\boldsymbol{t}) \in \mathscr{R}^{n} \\
\boldsymbol{D}\left(t_{0}\right) & =\boldsymbol{D}_{0}, \\
\dot{\boldsymbol{D}}\left(t_{0}\right) & =\boldsymbol{D}_{1}
\end{aligned}
$$

$I$ represents an open interval including $t_{0}$ and $f$ is a sufficiently smooth function on $I \times R^{n} \times R^{n}$. Then, $\boldsymbol{D}(t)$ is an analytic function that can be expressed by the following equation.

$$
\begin{aligned}
& \boldsymbol{D}(t)=\sum_{n=0}^{\infty} \frac{\boldsymbol{D}^{(n)}\left(t_{0}\right)}{n !}\left(t-t_{0}\right)^{n} \\
& \boldsymbol{D}(t)=\sum_{k=0}^{n} \frac{\boldsymbol{D}^{(k)}\left(t_{0}\right)}{k !}\left(t-t_{0}\right)^{k}+\boldsymbol{R}\left(n, t, t_{0}\right)
\end{aligned}
$$

The remainder term $\boldsymbol{R}\left(n, t, t_{0}\right)$ for the analytical series solution up to $\mathrm{n}$-degree term is explained and expressed as the following equation, and $c^{*}$, which satisfies the equation below, exists in the open interval. Moreover, the error is defined as expressed in the following equation (21).

$$
\begin{aligned}
& \boldsymbol{R}\left(n, t, t_{0}\right)=\frac{\boldsymbol{D}^{(n+1)}\left(c^{*}\right)}{(n+1) !}\left(t-t_{0}\right)^{n+1} \\
&\left|\boldsymbol{R}\left(n, t, t_{0}\right)\right| \leq \frac{1}{(n+1) ! t_{0} \leq t \leq t_{0}+t_{h}}\left|\boldsymbol{D}^{(n+1)}(t)\right| t_{h}{ }^{n+1}, \\
& t_{0} \leq t \leq t_{0}+t_{h}
\end{aligned}
$$

The Taylor series method defines the solution to (15) as ndegree series of (19) except for $\boldsymbol{R}$ term, and each differential coefficient, $\boldsymbol{D}^{(k)}\left(t_{0}\right)$, can be obtained from (15) to solve the equation. The computed solution has the precision with the error range of (21) in the defined interval of $\left[t_{0}, t_{0}+t_{h}\right]$. It is very efficient to solve the equation in multistep with $n$ and $t_{h}$ of (21) as the parameters. Adjusting for $n$ and $t_{h}$ is referred to variable order (VO) scheme and variable stepsize scheme, respectively. In particular, the two parameters $n$ and $t_{h}$ can determine the error limit, and the accuracy and computational speed of the solution are determined by them. This study defines the solution to (15) as finite Taylor series expressed in (22), and multistep approach is used for computing the solution.

$$
\begin{aligned}
\boldsymbol{D}_{i}(t)=\sum_{k=0}^{n} \frac{\boldsymbol{D}_{i}^{(k)}\left(t_{0 i}\right)}{k !}\left(t-t_{0 i}\right)^{k}, \\
\quad\left(t_{0 i} \leq t \leq t_{0 i}+t_{h}\right) i=1,2, \ldots, \text { total step }
\end{aligned}
$$

The initial value of $i$ step in the above equation can be found from previous step, and the differential coefficients, $\boldsymbol{D}^{(k)}\left(t_{0}\right)$, can be obtained from (15). Accordingly, an approximate analytical solution can be computed from the Taylor series at each step, and the number of terms increases with higher DOF (Degrees of Freedom) and higher order. This study used sufficient order $n$ and step-size $t_{h}$ to compute an accurate solution, and the process of (21) is not further discussed in this paper.

\section{Dynamic Snapping Model under Symmetric Mode}

Generally, the initiation of snapping process of shallow arches is differentiated by two different mechanisms. That is, one is direct snapping under symmetric mode as illustrated in Figure 2(a), and the other is indirect snapping under asymmetric mode as illustrated in Figure 2(b). In the figure, $\eta_{0}$ and $\eta_{i}$ are dimensionless initial configuration and dimensionless deflection from the initial configuration, respectively. First of all, symmetric deformation under sinusoidal distributed load is considered to deal with the solution for the case of direct snapping, and the coupling under asymmetric mode is discussed in the next chapter. Accordingly, when the 1st mode of deflection function is considered, (15) is developed into 2nd-order differential equation with respect to $D_{1}$ as expressed in the following equation.

$$
\ddot{D}_{1}+\gamma \dot{D}_{1}+\left(1+\frac{h^{2}}{2}\right) D_{1}-\frac{3}{4} h D_{1}{ }^{2}+\frac{1}{4} D_{1}{ }^{3}-\Lambda=0
$$

When the dimensionless time parameter $\tau$ is denoted by $\tau_{0}$ and the initial values are $D_{1}\left(\tau_{0}\right)=D_{0}$ and $\dot{D}_{1}\left(\tau_{0}\right)=\dot{D}_{0}$, the first two terms of the right-hand side become $D_{0}$ and $\dot{D}_{0}(\tau-$ $\left.\tau_{0 i}\right)$. Moreover, when the initial values are substituted into (23), the remaining differential coefficients up to $n$-degree term can be computed. The following equations show the coefficients of 3 rd and 4 th terms.

$$
\begin{aligned}
D_{1}{ }^{(2)}\left(\tau_{0}\right)= & -\gamma \dot{D}_{0}-\left(1+\frac{h^{2}}{2}\right) D_{0}+\frac{3}{4} h D_{0}{ }^{2}-\frac{1}{4} D_{0}{ }^{3} \\
& +\Lambda \\
D_{1}{ }^{(3)}\left(\tau_{0}\right)= & \gamma^{2} \dot{D}_{0}+\gamma\left(1+\frac{h^{2}}{2}\right) D_{0}-\frac{3}{4} \gamma h D_{0}{ }^{2} \\
& +\frac{1}{4} \gamma D_{0}{ }^{3}-\gamma \Lambda-\left(1+\frac{h^{2}}{2}\right) \dot{D}_{0} \\
& +\frac{3}{2} h D_{0} \dot{D}_{0}-\frac{3}{4} D_{0}{ }^{2} \dot{D}_{0}
\end{aligned}
$$




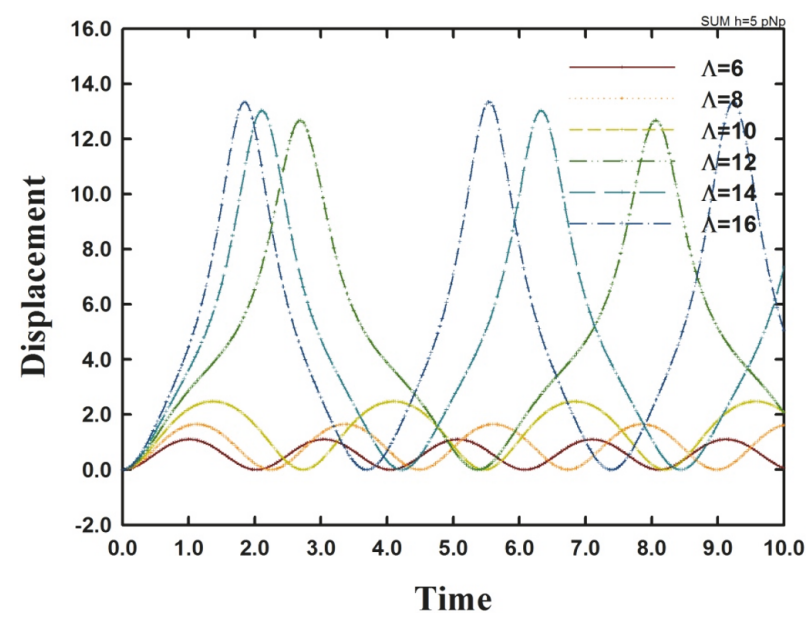

(a)

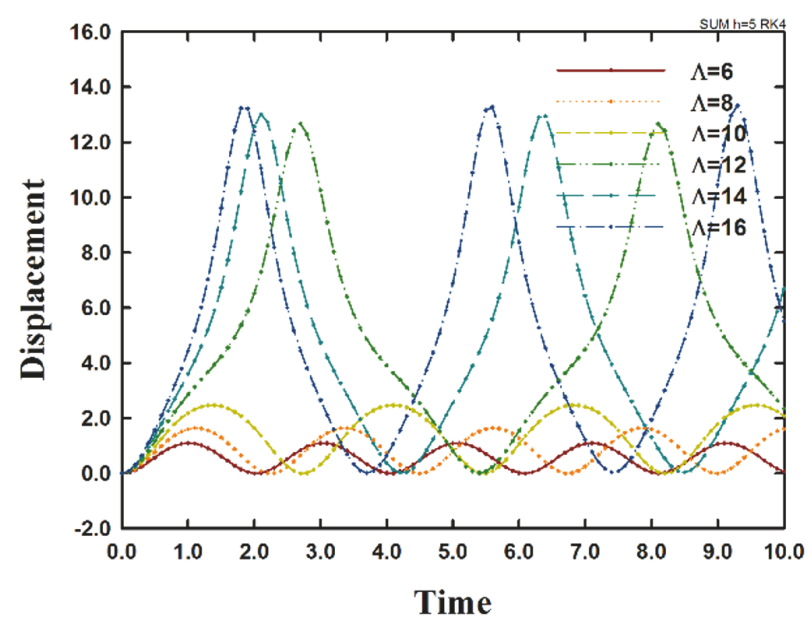

(b)

FiguRE 3: Displacement response of the shallow arches subjected to step excitation: (a) multistep Taylor series method and (b) 4th-order Runge-Kutta method (RK4).

Substituting these coefficients to (22) gives us the solution to the polynomial with respect to time, and the number of terms increases with degrees of freedom and order.

The appropriateness of the series solution to (23), which is composed of differential coefficients for explaining the dynamic snapping of shallow arches, is evaluated by applying step excitation. The solution was obtained by applying order $\mathrm{n}$ and step-size $t_{h}$ of 7 and 0.1, respectively, to the multistep Taylor series, and the time duration of the computation was set to be $100 \tau$.

Firstly, the analysis result with $\gamma=0$ (undamped system) is examined. Figure 3(a) shows the analysis result of step excitation with shape parameter $h$ of 5 , and it shows the displacement response of the shallow arches with the load level $\Lambda$ increasing from 6 to 16 in increment of 2 . Figures $3(\mathrm{a})$ and 3(b) are the results of the multistep Taylor series method and of the 4th-order Runge-Kutta method (RK4), respectively, for comparing the analysis results. In this case, the time parameters of the Taylor series method and RK4 were set at the same $t_{h}=0.1$. As shown in the figure, the results of the Taylor series method agree with the results of RK4. Besides, the figure shows that the nonlinear response is clearly differentiated in two groups of load levels (one group of $\Lambda=6.0,8.0$, and 10.0 and the other group of $\Lambda=12.0,14.0$, and 16.0). The first group had the increase in amplitude of the displacement and gradual lengthening of the period with the increase in the load level, but the second group exhibited conspicuous increase in displacement in comparison with the first group as well as shortening of the period. Moreover, the amplitude of the displacement did not vary linearly with the increase in $\Lambda$, and this is the effect of geometrical nonlinearity of the shallow arches. In other words, the former group represents prebuckling load level, and the latter group composes postbuckling load level.

Secondly, the maximum displacement response with $h=$ $1,3,5$, and 7 was examined in order to evaluate the solution to the dynamic behavior of the specimen in response to various shape parameter values, and the result is shown in Figure 4. In this figure, dynamic buckling load levels of $h=3,5$, and 7 correspond to $\Lambda=3.20,11.05$, and 27.9 , respectively. In particular, the figure shows that dynamic buckling (snapping) does not take place for the case of $h=1$, and the boundary point for the occurrence of buckling is higher with higher $h$.

The attractor in phase plan as shown in Figure 5 was such that the orbit changed from the form of one drop of water to the form of two drops of water linked together after reaching dynamic snapping load level, and this well explains the dynamic unstable phenomenon of the shallow arches.

To explain why snapping does not take place for the case of $h=1$, there is a need to investigate the equilibrium points of the shallow arch system, and their stability. Let $D_{1}=u-h$ and $\dot{u}=v$. Equation (23) can then be written as the 1st-order system, as shown in (26). For this system, the equilibrium points are obtained by solving the system $v=0$ and $\dot{v}=0$, and the stability of the points can be observed based on the characteristic polynomial of the Jacobian matrix.

$$
\begin{aligned}
& \dot{u}=v \\
& \dot{v}=-\gamma v-\left\{\frac{1}{4} u^{3}+\left(1-\frac{h^{2}}{4}\right) u-h-\Lambda\right\}
\end{aligned}
$$

In the case of this system, the equilibrium points and their stability were studied by $\mathrm{Ha}$ et al. [18], and they can be summarized as follows.

The problem of finding the equilibrium points can be reduced to the investigation of the roots of the 3rd-order polynomial equation as follows:

$$
u^{3}+\left(4-h^{2}\right) u=4(h+\Lambda)
$$

Let $f(u)=u^{3}+\left(4-h^{2}\right) u=u\left[u^{2}+\left(4-h^{2}\right)\right]$, and there are two cases depending on $h$. The first case is for $|h| \leq 2$. The equation has only one real root, and the sign is equal to $(h+\Lambda)$. The second case is for $|h|>2$. Then $f^{\prime}\left(u_{0}\right)=0$ for 


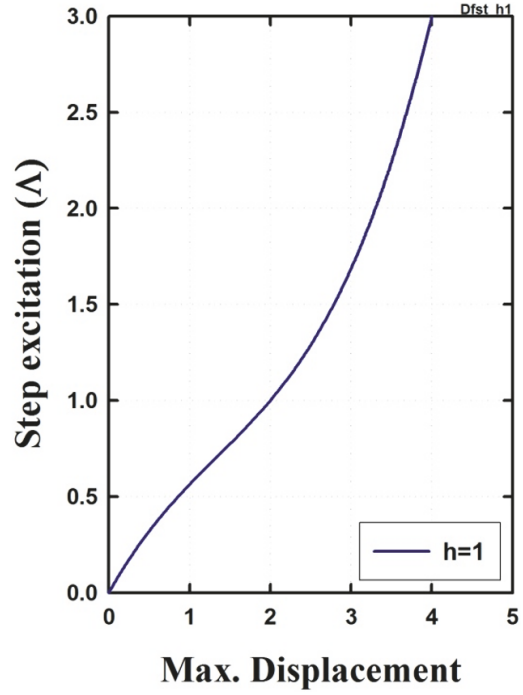

(a)

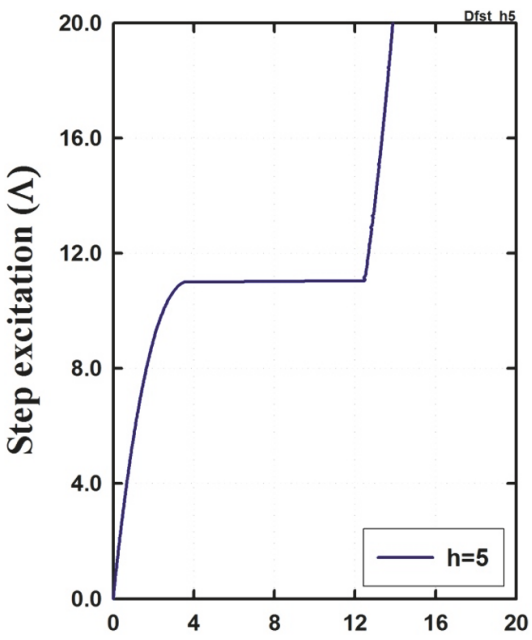

Max. Displacement

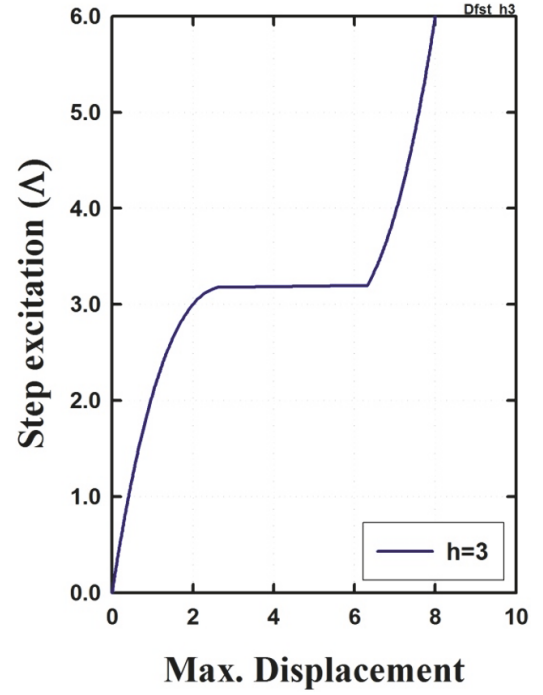

(b)

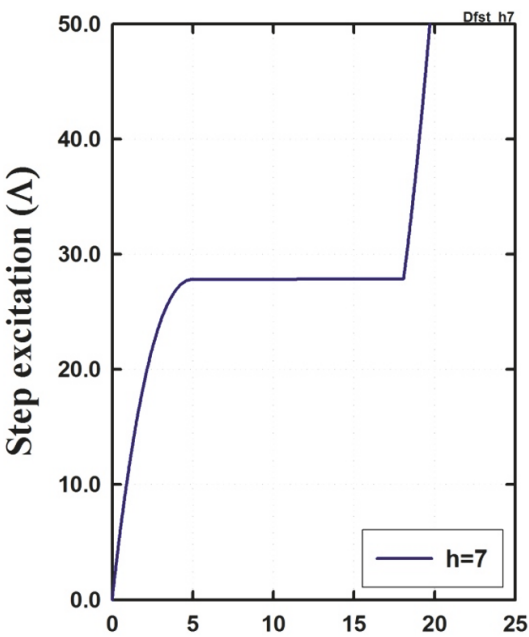

Max. Displacement

(d)

FIgURE 4: Maximum displacement curves under step excitation: (a) $h=1$; (b) $h=3$; (c) $h=5$; and (d) $h=7$.

$u_{0}=\sqrt{\left(h^{2}-4\right) / 3}$. The equation has a local maximum $f\left(-u_{0}\right)$ and minimum $f\left(u_{0}\right)$, as shown in $(28)$, and the number of equilibria and their signs depend on $(h+\Lambda)$. For instance, it has a positive root and two negative roots for $0<4(h+\Lambda)<$ $f\left(-u_{0}\right)$.

$$
f\left(\mp \mathrm{u}_{0}\right)= \pm \frac{2 \sqrt{3}}{9}\left(h^{2}-4\right)^{3 / 2}
$$

From analyzing the characteristic polynomial of the Jacobian matrix, the stability of the equilibrium point $(\widehat{u}, 0)$, where $\widehat{u}$ is a root of (26), can be defined as follows:

(i) Case $|h| \leq 2$ : unique equilibrium $(\widehat{u}, 0)$ is asymptotically stable.

(ii) Case $|h|>2$ : for $4|h+\Lambda|>f\left(-u_{0}\right)$, equilibrium $(\widehat{u}, 0)$ is asymptotically stable. For $4|h+\Lambda|<f\left(-u_{0}\right)$, there are two asymptotically stable points $(\widehat{u}, 0)$ satisfying $|\widehat{u}|>u_{0}$, and one unstable point $(\widehat{u}, 0)$ satisfying $|\widehat{u}|<$ $u_{0}$. For $4|h+\Lambda|=f\left(-u_{0}\right)$, there are two equilibrium points: stable point $\left(-u_{0}, 0\right)$ and asymptotically stable point $(\widehat{u}, 0)$ with $\widehat{u}>u_{0}$.

As regards the condition of $h>0$ and $\Lambda \leq 0$, which means acting on the negatively vertical direction, Figure 6 shows the stability of the equilibrium points under this condition. If $h \leq 2$ and $\Lambda=0$, then the equilibrium point is the unique root of $f(u)=4 h$ and it is asymptotically stable. In spite of the increase in the magnitude of $|\Lambda|$, the point is still asymptotically stable (see Figure 6(a)). If $h>2$ and $\Lambda=0$, the stability depends on the relationship between $4 h$ and $f\left(-u_{0}\right)$. There are three cases: $2<h<4, h=4$, and $h>4$. For the first case, the equilibrium point under excitation $\Lambda=0$ started an asymptotically stable point (see Figure 6(b)). For 


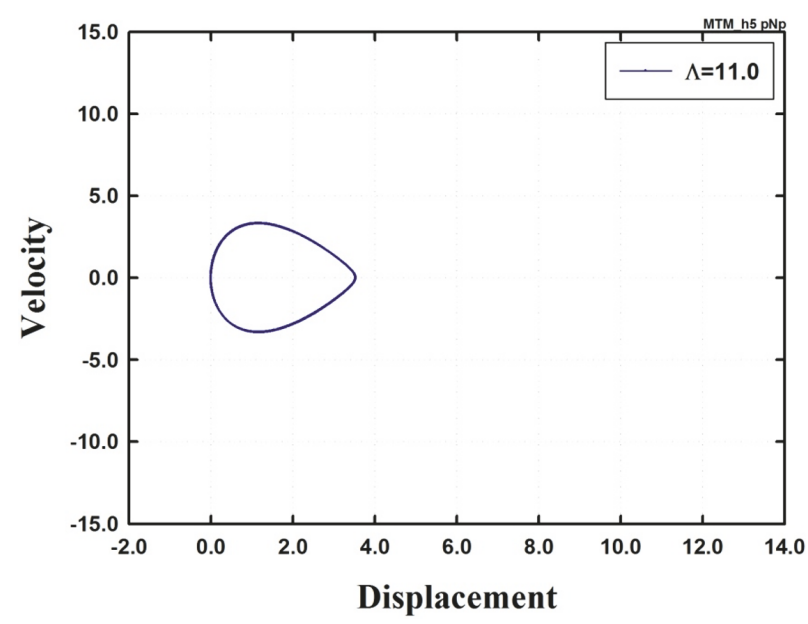

(a)

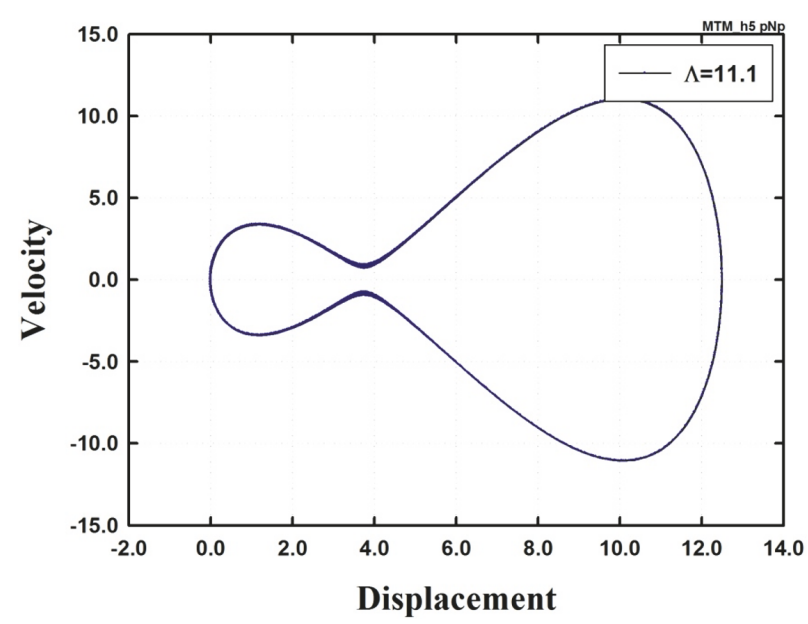

(b)

FIGURE 5: Phase diagram of the vertical motion for shape parameter $h=5$ : (a) trajectory of prebuckling load level, $\Lambda=11.0$; and (b) trajectory of postbuckling load level, $\Lambda=11.1$.

the next case, a stable point and an asymptotically stable point are observed (see Figure 6(c)). For the final case, two asymptotically stable points and an unstable point are started (see Figure $6(\mathrm{~d})$ ). As the $|\Lambda|$ increased for these cases, the stabilities are varied, as shown in Figures 6(b) $-6(d)$. As shown in Figure 6 , the case of $h \leq 2$ has only a unique asymptotically stable point, and it can be expected that snapping will not take place. On the other hand, the case of $h>4$ started two asymptotically stable points and one unstable point, and it can be expected that the system is very sensitive.

Next, the result of considering the effect of damping coefficient is shown in Figure 7, and it shows that the displacement converges faster with greater $\gamma$. The attraction shown in Figure 7(b) deviates from the trajectory of the first form of a drop of water and converges gradually to the water drop at the opposite side. This indicates that the trajectory becomes stable state asymptotically. In this model, the shape parameter $h$ and load level $\Lambda$ are 5 and 11.3, respectively, with damping coefficients of $\gamma=0.0,0.02$, and 0.05 .

As the arches may violently vibrate around critical buckling level $\Lambda$ and at low $\gamma$, there is a need to investigate the equilibrium values about $(\gamma, \Lambda)$, which is plotted in Figure 8. Figures 8(a) and 8(b) are for $h=3$ and $h=5$, respectively, and the equilibrium values are computed. The boundaries of this map are $(\gamma, \Lambda) \in[-4.5,-2.5] \times(0,0.5]$ for $h=3$ and $(\gamma, \Lambda) \in[-15,-10] \times(0,0.5]$ for $h=5$, respectively. In the figures, the red points correspond to $u>0$, and the blue points correspond to $u<0$. The figures show that the sign of $u$ is clearly separated along the critical values when $\gamma>0.1$, and there appears a mixed critical boundary around $[-4,-3] \times$ $(0,0.1]$ for $h=3$ and around $[-14,-11] \times(0,0.1]$ for $h=5$. These results show that the lower damping coefficient $\gamma$ is, the more sensitive the variation around the critical boundary will be.

Finally, consider the stability of the arches in accordance with the different initial values. In particular, the equilibrium values about $\left(u_{\tau=0}, v_{\tau=0}\right)$, which is plotted in Figure 9, are investigated. $\left(u_{\tau=0}, v_{\tau=0}\right)$ is the initial value of the 1st-order system, as shown in (26). The damping oscillation with $\gamma=$ 0.2 and $\Lambda=0$ is analyzed, and the equilibrium values with $h=1,3,5$, and 7 are computed. In the figure, plane element $\left(u_{\tau=0}, v_{\tau=0}\right) \in[-20,20] \times[-20,20]$ is divided into $250 \times$ 200 elements as the initial values on the lattice point of the coordinate and time $\tau \in[0,200]$.

As shown in Figure 6, the equilibrium points are distinguished by the range of $h$. For $h<4$ and $\Lambda=0$, there is a unique stable point, and when the time increases from the initial value $\left(u_{\tau=0}, v_{\tau=0}\right), u(\tau)$ focuses on a single point, as shown in Figures 9(a) and 9(b). For $h>4$ and $\Lambda=0$, there are two asymptotically stable points and an unstable point, and when the time increases from the initial value $\left(u_{\tau=0}, v_{\tau=0}\right)$, $u(\tau)$ focuses on the two stable points, as shown in Figures 9 (c) and $9(\mathrm{~d})$. If the trajectory is attracted to the equilibrium points, the lattice element of the initial value is drawn in red, otherwise, in blue. For Figures 9(a) and 9(b), red is almost equal to blue, but Figures 9(c) and 9(d) have different signs and magnitudes.

For better understanding, the extended phase diagrams are drawn in Figure 10. These results can be easily observed from Figures 10(c) and 10(d). From the results of $h=5$ and $h=7$, the boundary of the color becomes the boundary of the attractor. In particular, a pair of insets can be clearly seen through the equilibrium values, and these points are divided into positive and negative values.

In conclusion, the analytical series solution converged even when damping was considered, and it could well predict the phenomenon and characteristics of the expected direct snapping of shallow arches.

\section{Dynamic Snapping Model under Asymmetric Mode}

This chapter describes indirect snapping model due to coupling in symmetric mode and asymmetric mode. Since the 


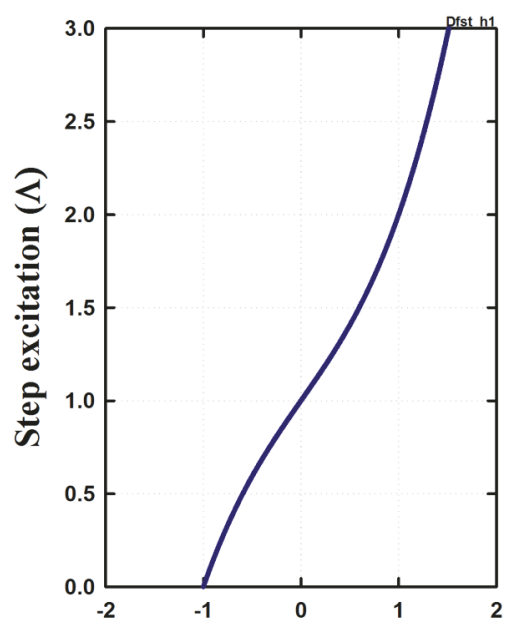

Equilibrium point $(\widehat{u})$

— Asymptotically stable

(a)

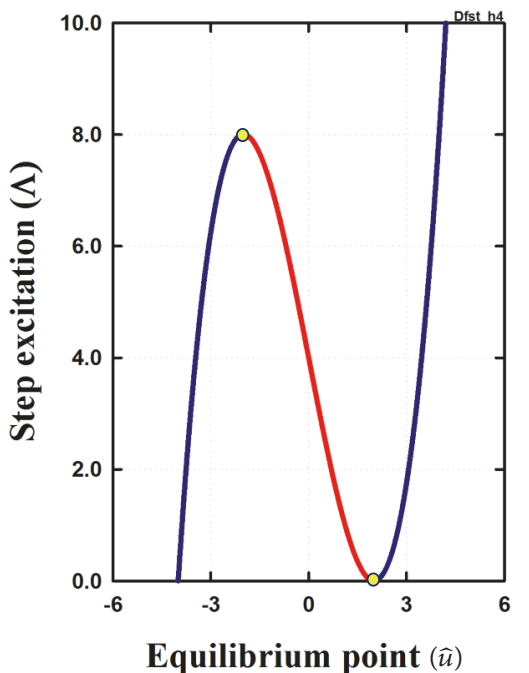

_ Asymptotically stable
_ Unstable
Stable

(c)

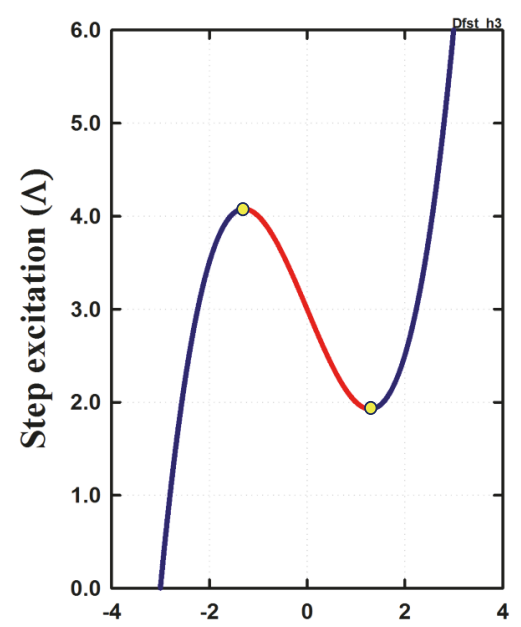

Equilibrium point $(\widehat{u})$

_ Asymptotically stable
Unstable
Stable

(b)

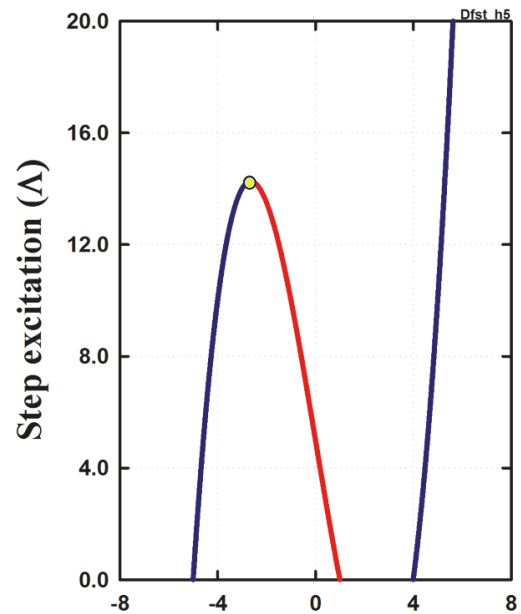

Equilibrium point $(\widehat{u})$

— Asymptotically stable
_ Unstable
Stable

(d)

FIGURE 6: Equilibrium points under the step excitations: (a) $h=1$; (b) $h=3$; (c) $h=4$; and (d) $h=5$.

influence of asymmetric mode as shown in Figure 2(b) should consider both the first term $D_{1}$ and second term $D_{2}$ of displacement function, the following 2nd-order differential equations with respect to $D_{1}$ and $D_{2}$ are derived from (15).

$$
\begin{aligned}
& \ddot{D}_{1}+\gamma \dot{D}_{1}+\left(1+\frac{1}{2} H^{2}\right) D_{1}-\frac{3}{4} H D_{1}{ }^{2}-H D_{2}{ }^{2} \\
& \quad+D_{1} D_{2}{ }^{2}+\frac{1}{4} D_{1}^{3}-\Lambda=0 \\
& \ddot{D}_{2}+\gamma \dot{D}_{2}+16 D_{2}-2 H D_{1} D_{2}+D_{1}{ }^{2} D_{2}+4 D_{2}^{3}=0
\end{aligned}
$$

When $\tau=\tau_{0}$ and the initial values are set to be $D_{1}\left(\tau_{0}\right)=D_{1}^{0}$, $\dot{D}_{1}\left(\tau_{0}\right)=\dot{D}_{1}^{0}, D_{2}\left(\tau_{0}\right)=D_{2}^{0}$, and $\dot{D}_{2}\left(\tau_{0}\right)=\dot{D}_{2}^{0}$ as described in the previous chapter, the first two terms of the power series solution are $D_{1}^{0}$ and $\dot{D}_{1}^{0}\left(\tau-\tau_{0 i}\right)$ for the case of $D_{1}$ and $D_{2}^{0}$ and $\dot{D}_{2}^{0}\left(\tau-\tau_{0 i}\right)$ for the case of $D_{2}$. The remaining terms are expressed in the following when the order $n$ is 4 as mentioned in the previous chapter.

$$
\begin{aligned}
D_{1}^{(2)}\left(\tau_{0}\right)= & -\gamma \dot{D}_{1}^{0}-\left(1+\frac{h^{2}}{2}\right) D_{1}^{0}+\frac{3}{4} h D_{1}^{0^{2}}+h D_{2}^{0^{2}} \\
& -D_{1}^{0} D_{2}^{0^{2}}-\frac{1}{4} D_{1}^{0^{3}}-\Lambda
\end{aligned}
$$




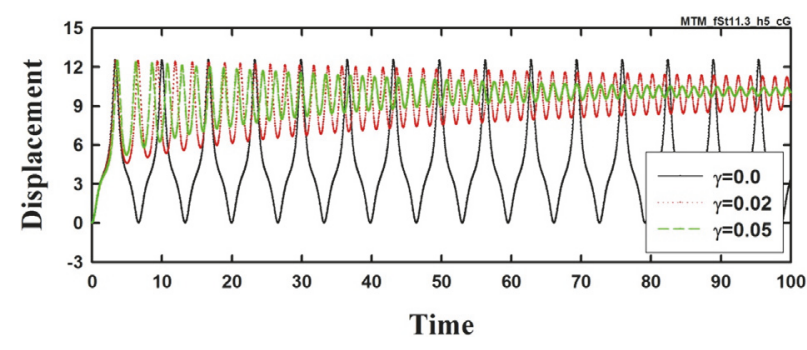

(a)

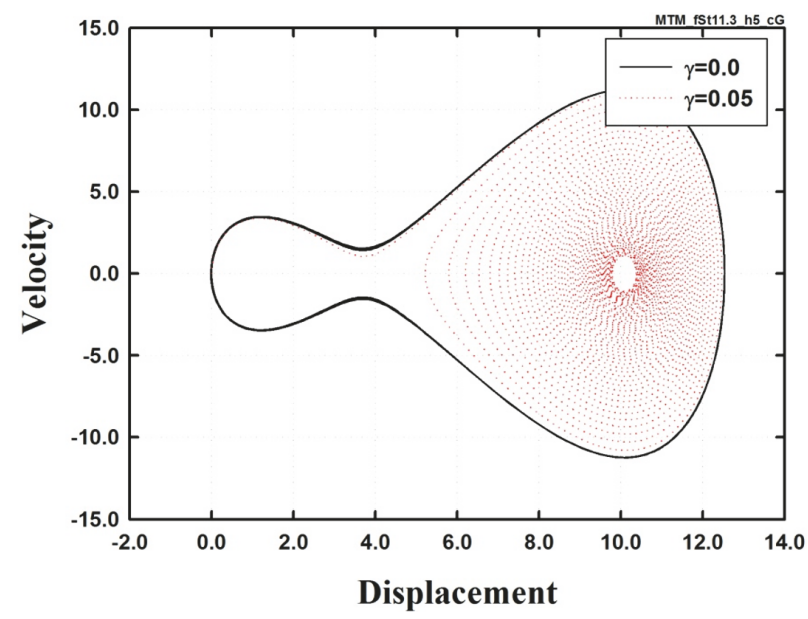

(b)

FIGURE 7: Results of the dynamic analysis in consideration of damping coefficient $\gamma$ under step excitation $\Lambda$ : (a) transient response of the motion and (b) trajectory in phase plan.

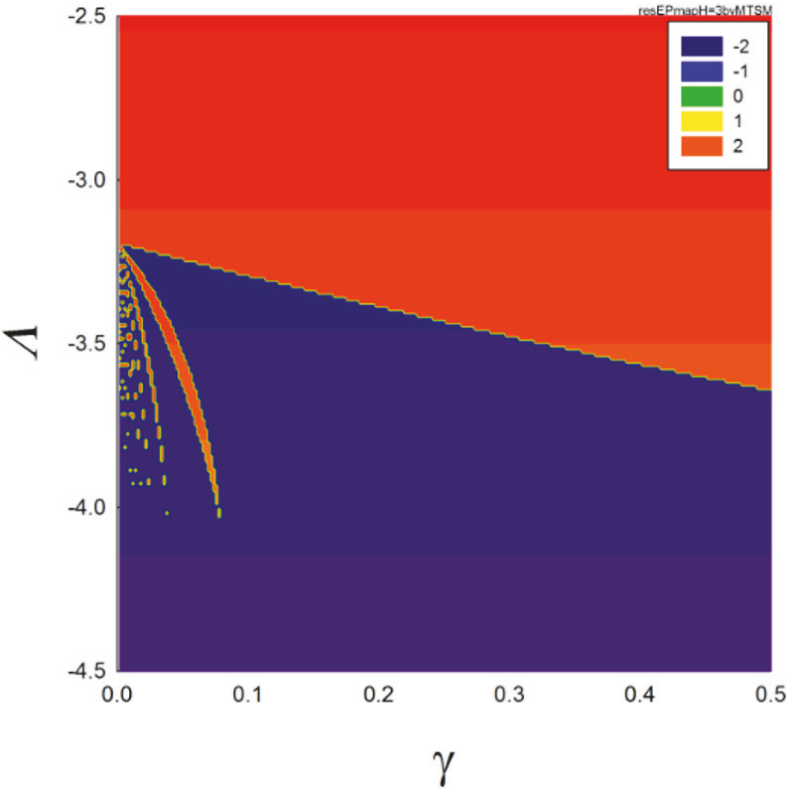

(a)

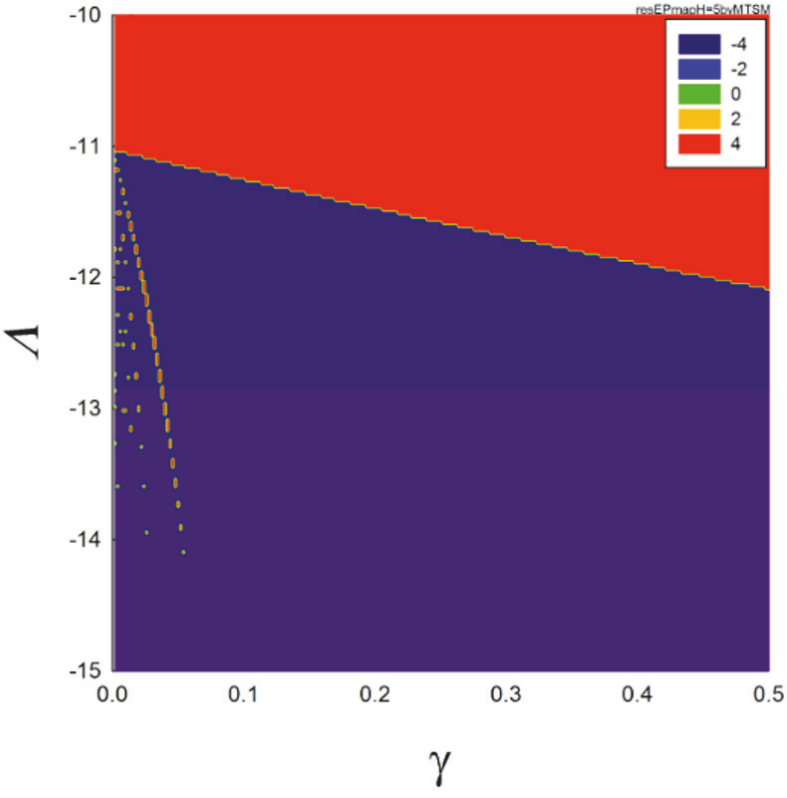

(b)

FIGURE 8: Equilibrium points and critical buckling boundary in accordance with the change in the damping coefficient: (a) $h=3$ and (b) $h=5$.

$$
\begin{aligned}
D_{1}^{(3)}\left(\tau_{0}\right)= & \gamma^{2} \dot{D}_{1}^{0}+\gamma\left(1+\frac{h^{2}}{2}\right) D_{1}^{0}-\frac{3}{4} \gamma h D_{1}^{0^{2}} \\
& -\gamma h D_{2}^{0^{2}}+\gamma D_{1}^{0} D_{2}^{0^{2}}+\frac{1}{4} \gamma D_{1}^{0^{3}}-\gamma \Lambda \\
& -\left(1+\frac{h^{2}}{2}\right) \dot{D}_{1}^{0}+\frac{3}{2} h D_{1}^{0} \dot{D}_{1}^{0}-D_{2}^{0^{2}} \dot{D}_{1}^{0} \\
& -\frac{3}{4} D_{1}^{0^{2}} \dot{D}_{1}^{0}+2 h D_{2}^{0} \dot{D}_{2}^{0}-2 D_{1}^{0} D_{2}^{0} \dot{D}_{2}^{0}
\end{aligned}
$$$$
D_{2}^{(2)}\left(\tau_{0}\right)=-\gamma \dot{D}_{2}^{0}-16 D_{2}^{0}+2 h D_{1}^{0} D_{2}^{0}-D_{1}^{0^{2}} D_{2}^{0}
$$

$$
\begin{aligned}
D_{2}{ }^{(3)}\left(\tau_{0}\right)= & \gamma^{2} \dot{D}_{2}^{0}+16 \gamma D_{2}^{0}-2 \gamma h D_{1}^{0} D_{2}^{0}+\gamma D_{1}^{0^{2}} D_{2}^{0} \\
& +4 \gamma D_{2}^{0^{3}}+2 h D_{2}^{0} \dot{D}_{1}^{0}-2 D_{1}^{0} D_{2}^{0} \dot{D}_{1}^{0} \\
& -16 \dot{D}_{2}^{0}+2 h D_{1}^{0} \dot{D}_{2}^{0}-D_{1}^{0^{2}} \dot{D}_{2}^{0} \\
& -12 D_{2}^{0^{2}} \dot{D}_{2}^{0}
\end{aligned}
$$




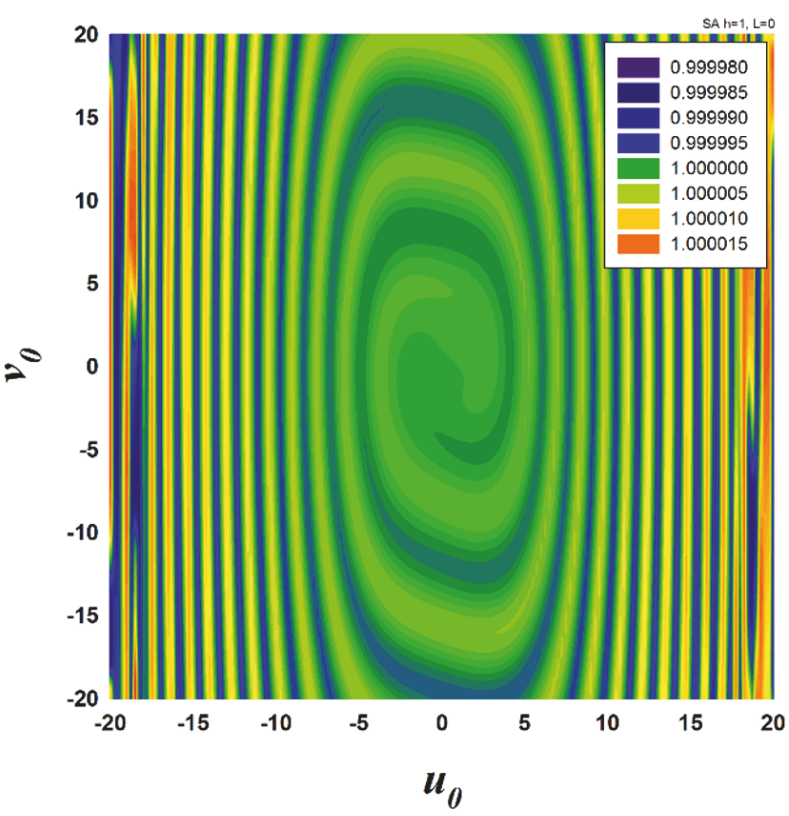

(a)

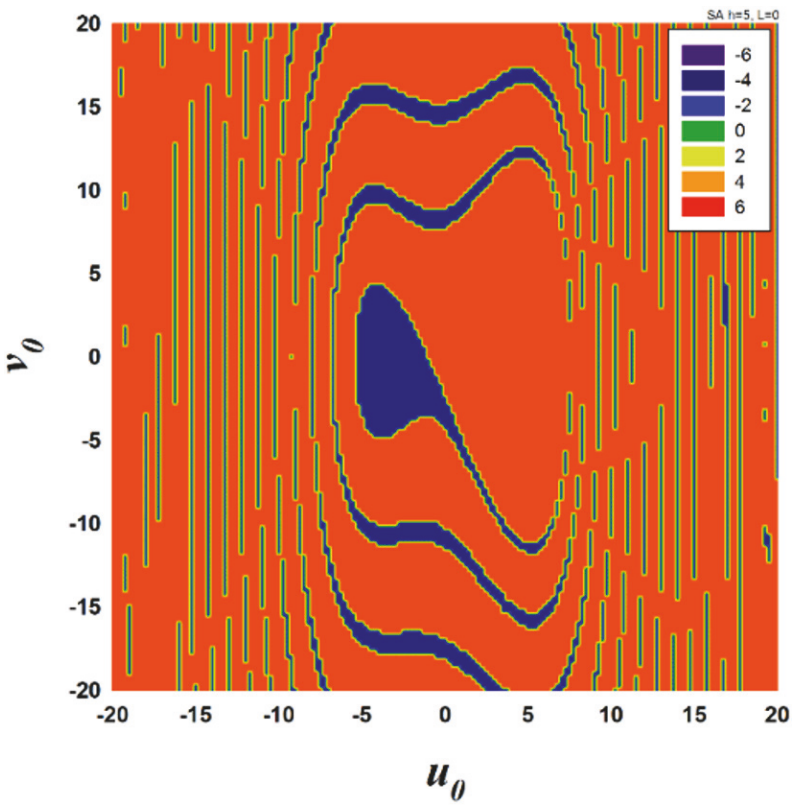

(c)

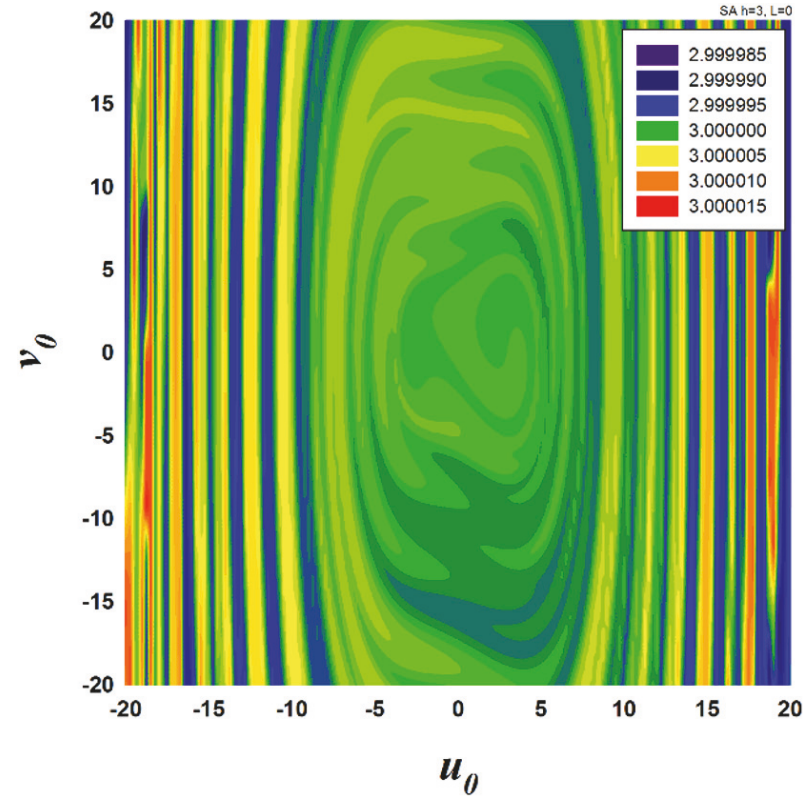

(b)

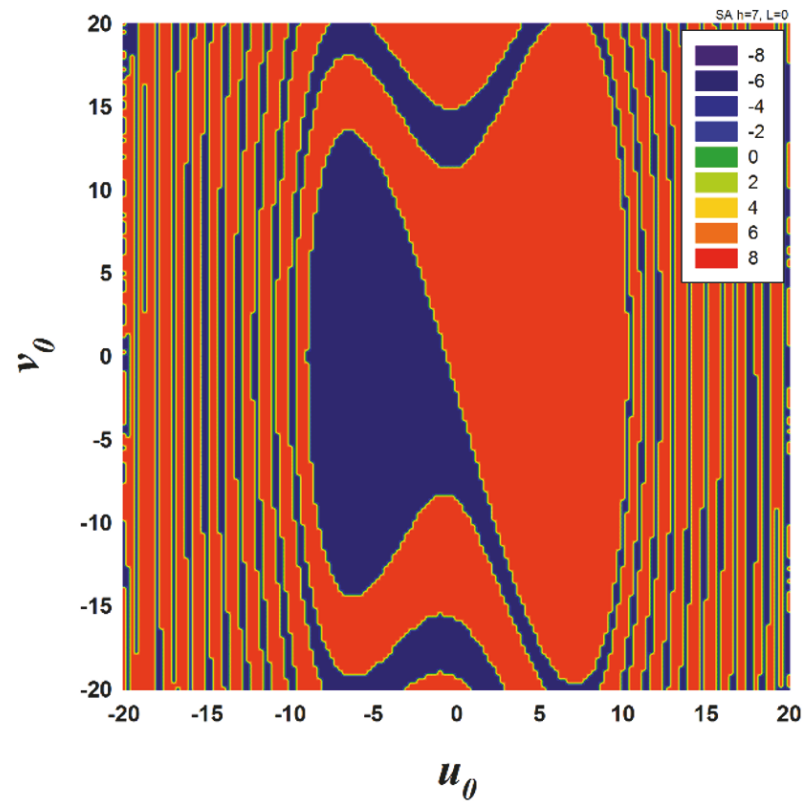

(d)

FIGURE 9: Attraction of the 1st-order system of arches under excitation level $\Lambda=0$ and damping coefficient $\gamma=0.2$ : (a) $h=1$; (b) $h=3$; (c) $h=5$; and (d) $h=7$.

The analysis continued to investigate the result of the solution to indirect snapping for the shape of $h$ with parameters of $n=7$ and $t_{h}=0.1$. In this analysis, $0.1 \%$ of $h$ was applied to account for the initial imperfection, and beating excitation and step and periodic excitation was carried out.

Figure 11 is the displacement response to step excitation for load level $\Lambda=18.5$ and shape parameter $h=7$. In case of Figure 11(a), imperfection is not considered, but it is introduced by $D_{2}=0.001 h$, i.e., $0.1 \%$ of $h$, in case of Figure 11(b). Figure 11(b) in consideration of initial imperfection shows that displacement rapidly changes due to the influence of coupling under asymmetric mode past the time level of 10. This is also observed in Figure 12, showing the change of attraction in the phase plan. Considering the boundary of dynamic critical load level for direct snapping, $\Lambda=27.9$, the result of Figure 11 indicates that the shallow arch test specimens are very sensitive to initial condition.

To verify and compare with the analysis results by multistep Taylor series method, the models in Figure 11 are recomputed using $\mathrm{RK} 4$, and the results are shown in 


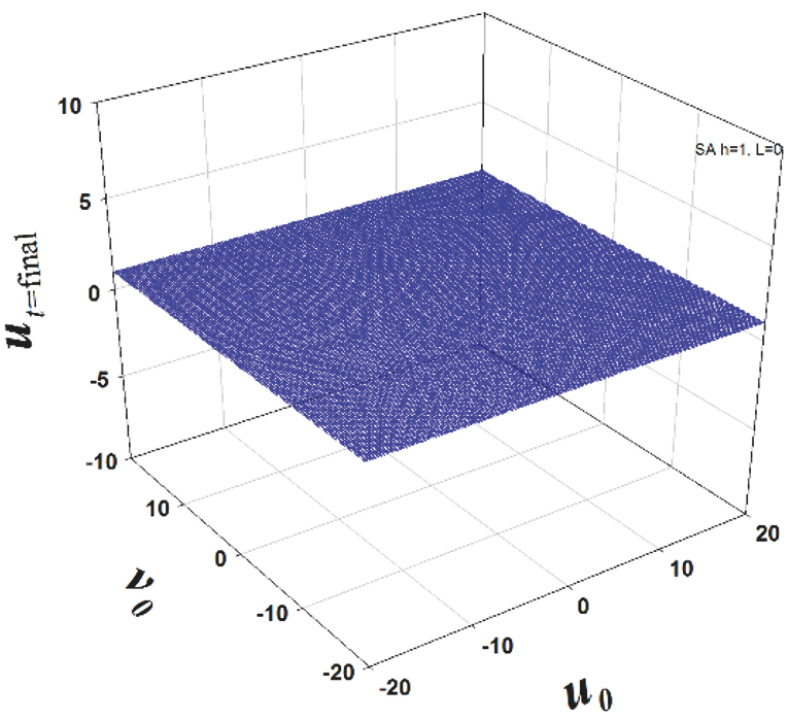

(a)

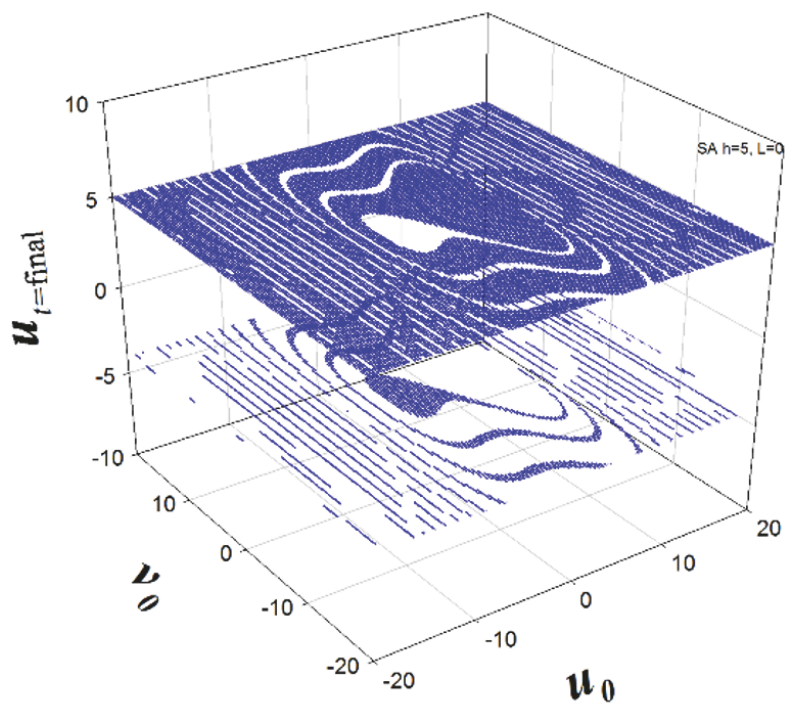

(c)

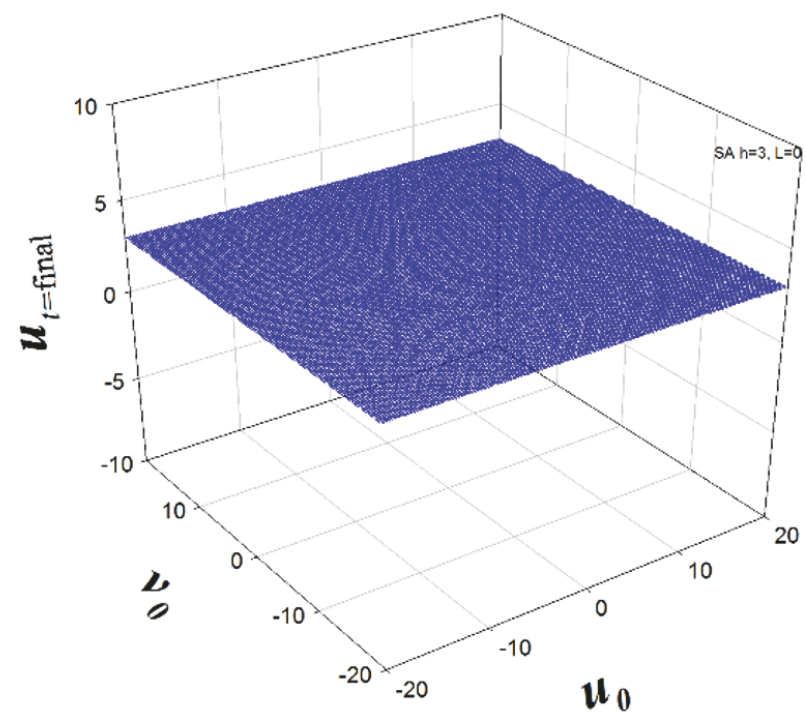

(b)

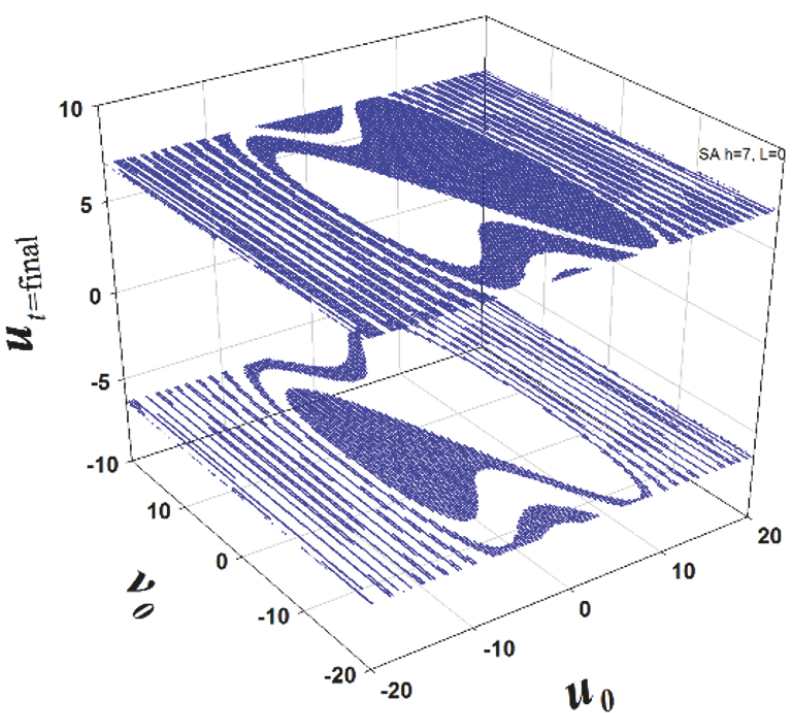

(d)

Figure 10: Expended phase space of the 1st-order system of arches: (a) $h=1 ;$ (b) $h=3$; (c) $h=5$; and (d) $h=7$.

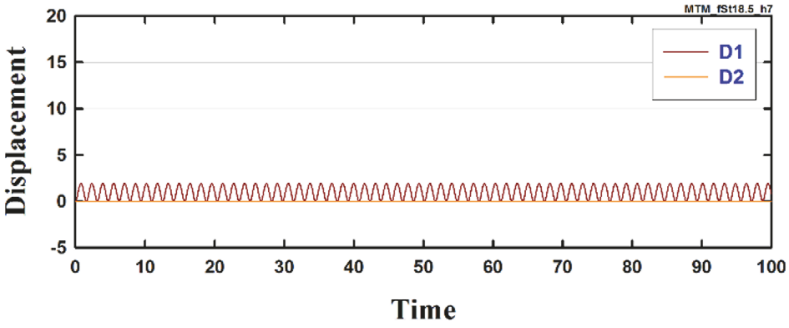

(a)

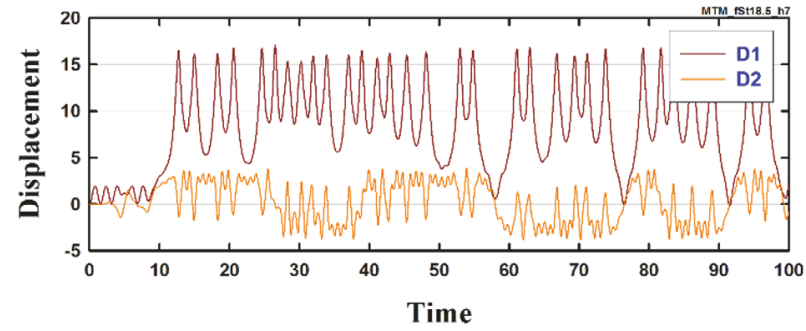

(b)

FIGURE 11: Transient response of shallow arches with initial imperfection $\left(t_{h}=0.1\right)$ (MTSM): (a) perfection, $D_{2}=0.0$; and (b) initial imperfection $D_{2}=0.001 \mathrm{~h}$. 


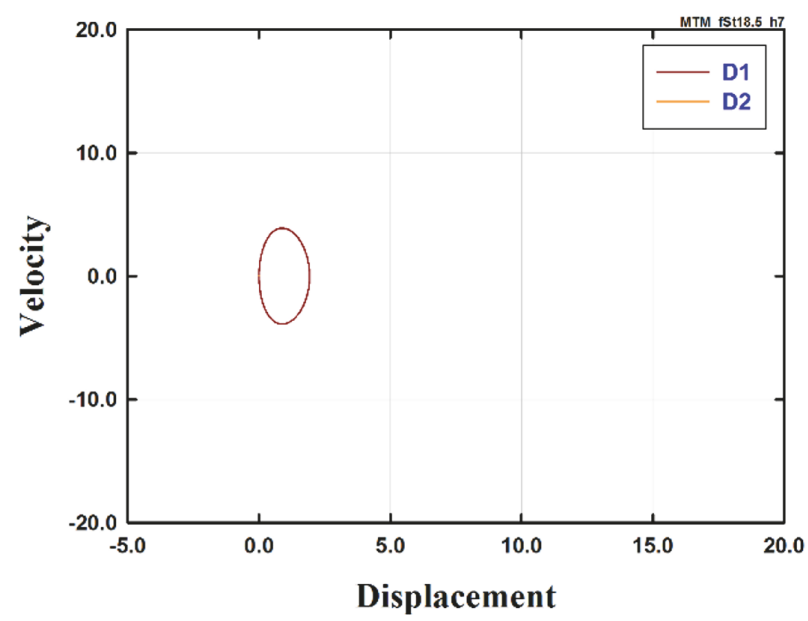

(a)

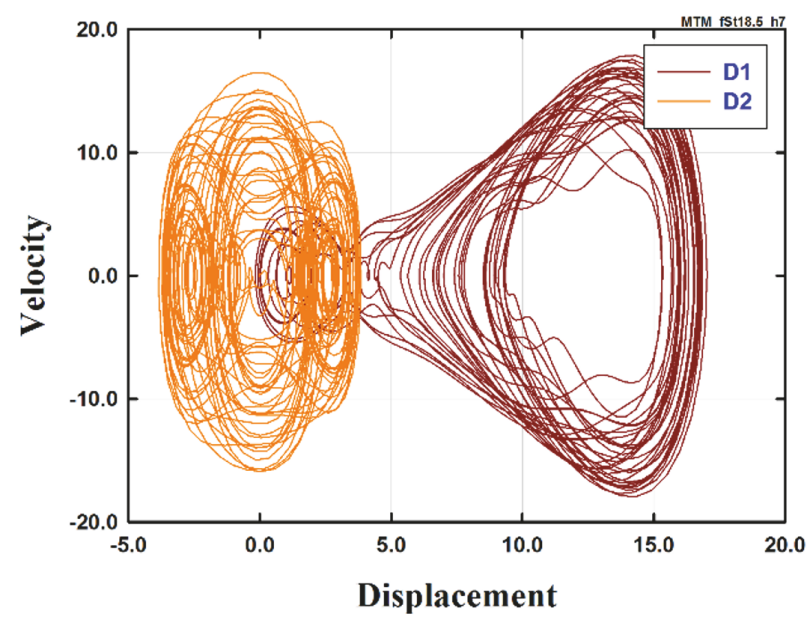

(b)

FIGURE 12: Phase diagram of the dynamic response of the analytical model with initial imperfection $(h=7, \Lambda=18.5)($ MTSM): (a) trajectory of $D_{2}=0.0$ and (b) trajectory of $D_{2}=0.001 h$.

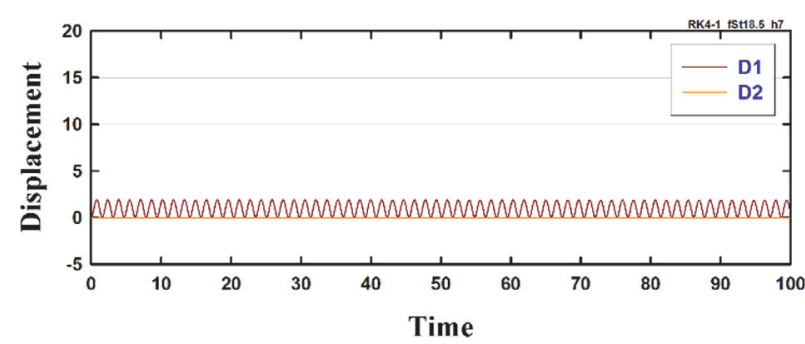

(a)

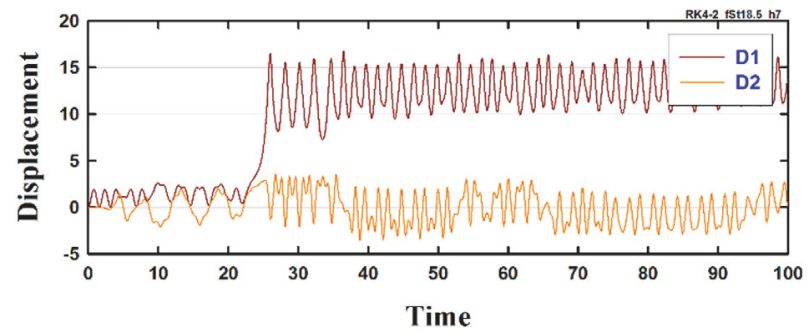

(b)

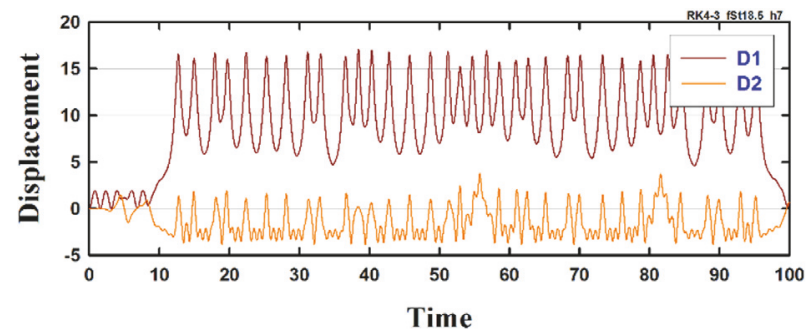

(c)

FIgURE 13: Transient response of the shallow arches with initial imperfection (RK4): (a) perfection $D_{2}=0.0, t_{h}=0.1$; (b) initial imperfection $D_{2}=0.001 h, t_{h}=0.1$; and (c) initial imperfection $D_{2}=0.001 h, t_{h}=0.001$.

Figures 13 and 14. In the case of the perfect model, the time parameters of the Taylor series method and RK4 were set at the same $t_{h}=0.1$. As shown in Figures 11(a) and 13(a), the results of the Taylor series method agree with the results of RK4. Besides, the trajectory in Figure 12(a) well matches the trajectory in Figure 14(a).

Considering the imperfection model in Figure 11(b), the results of RK4 corresponding to this imperfection model are shown in Figures 13(b) and 13(c), which are for $t_{h}=0.1$ and for $t_{h}=0.001$, respectively. From the result shown in Figure 13(b), the response pattern is similar, but it can be seen that they do not coincide. On the other hand, Figure 13(c) is more consistent than Figure 13(b). As the imperfection model is more sensitive than the perfection model, a small time internal $t_{h}$ is required for RK4. Besides, the results pattern can be easily observed from Figures 14(b) and 14(c). The results shown in Figures 13 and 14 indicate that the shallow arches are very sensitive to the initial condition, and higher accuracy is required to analyze the sensitive model.

Multistep Taylor series were applied to the analysis results of sinusoidal excitation and beating excitation as shown in Figure 15. The periodic parameters $\alpha$ and $\beta$ of 1.0 and 0.05 , respectively, were applied as shown in Figure 15.

$$
\begin{aligned}
& \Lambda=\Lambda_{0} \cdot \sin \left(\alpha w_{0} \tau\right) \\
& \Lambda=\Lambda_{0} \cdot 0.5\left\{\cos \left(\alpha w_{0} \tau\right)-\cos \left(\alpha(1-\beta) w_{0} \tau\right)\right\}
\end{aligned}
$$




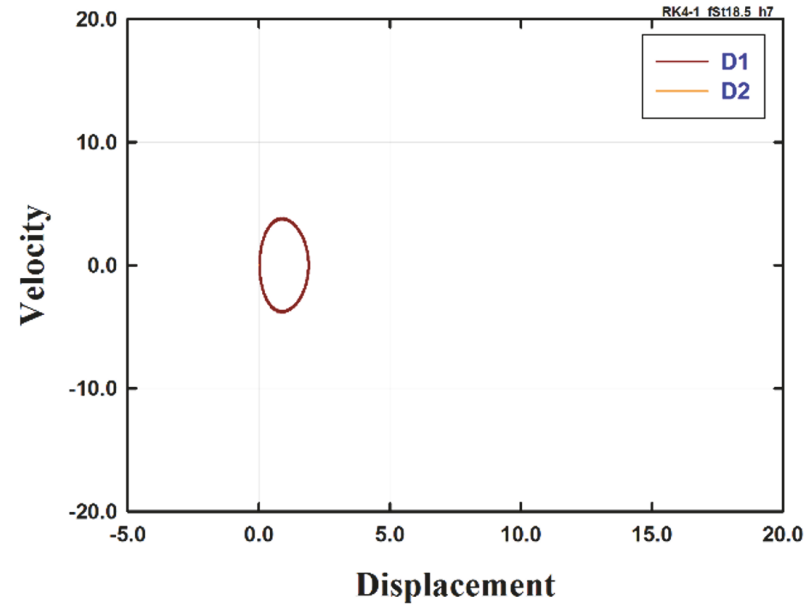

(a)

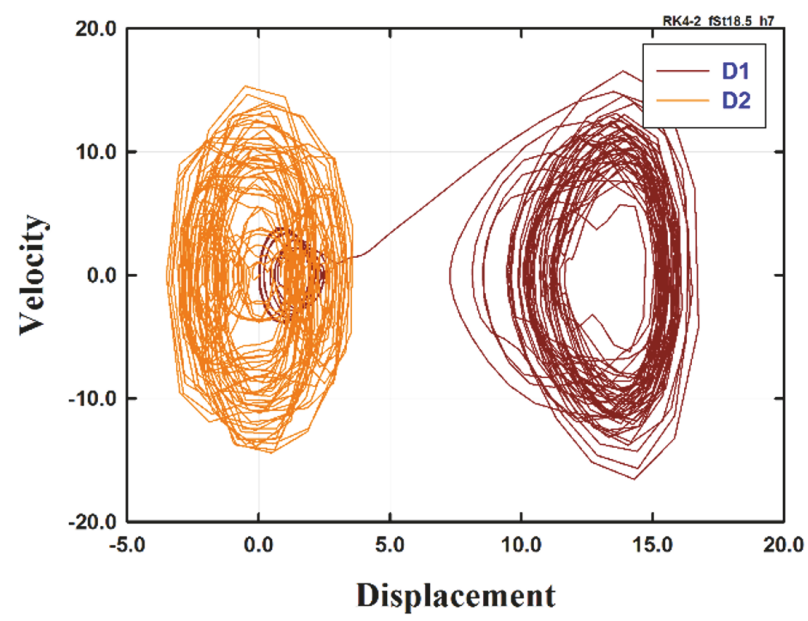

(b)

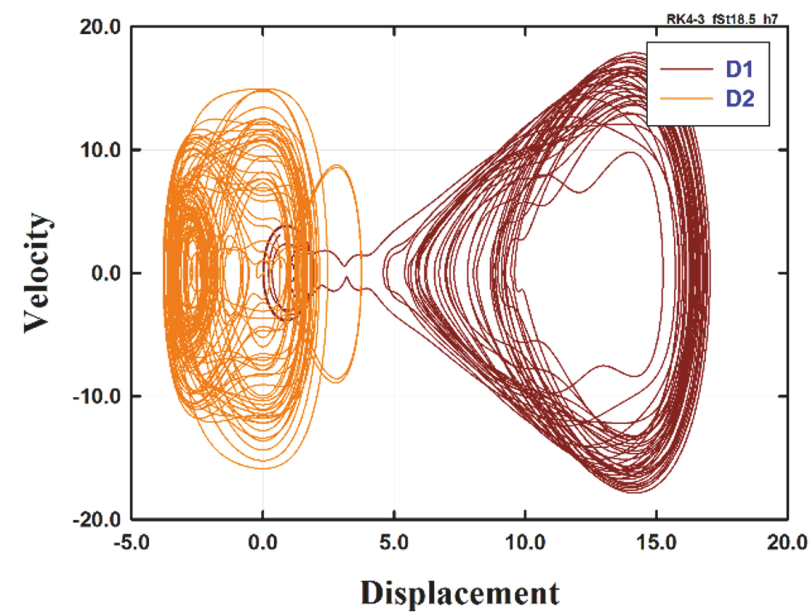

(c)

FIGURE 14: Phase diagram of the dynamic response of the analytical model with initial imperfection $(h=7, \Lambda=18.5)($ RK4): (a) perfection $D_{2}=0.0, t_{h}=0.1 ;(\mathrm{b})$ initial imperfection $D_{2}=0.001 h, t_{h}=0.1$; and (c) initial imperfection $D_{2}=0.001 h, t_{h}=0.001$.

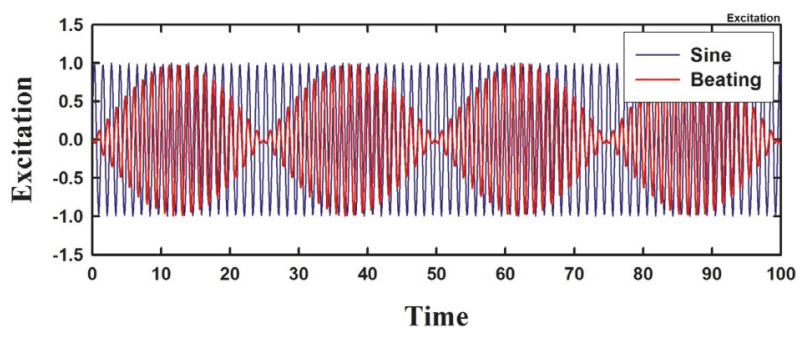

FIGURE 15: Sinusoidal and beating excitations.

In (34) and (35), $w_{0}$ is the first natural angular frequency of the model, and the excitation is applied at the same period as the natural frequency of the case of $\alpha=1.0$.

This study considered the case of $\alpha=1.0$ only. Accordingly, since resonance was expected by excitation, the analysis continued with lower level $\Lambda_{0}$ of 8.0 than the buckling load level under step excitation. Figure 16 shows the analysis result. In this example, the shape parameter and load level of sinusoidal excitation are $h=7$ and $\Lambda_{0}=8.0$, respectively. Imperfection is not considered in Figure 16(a), but it is introduced by $D_{2}=0.001 h$, i.e., $0.1 \%$ of $h$ in case of Figure 16(b). After the time elapses to 20 as shown in figure (b), the amplitude of symmetric mode $D_{1}$ is increased by the perturbation of the asymmetric mode $D_{2}$. It indicates that, for the case of sinusoidal excitation without the consideration of imperfection (Figure 16(a)), there was no rapid increase in the observed displacement. Nevertheless, the effect of coupling was very great for the case of considering asymmetric mode (Figure 16(b)). This result is manifested clearer with the trajectory in phase plan as shown in Figure 17. In other words, although there was no change of trajectory in the phase plan as shown in Figure 17(a), Figure 17(b) shows that the $D_{1}$ curve changes the form of trajectory after the elapse of a certain time.

The analysis of beating excitation was carried out with $h=7$ like the case of sinusoidal excitation, and $\Lambda_{0}$ of 8.0, the same load level as for sinusoidal excitation, was applied. Figure 18 shows the analysis result. After the time elapses 


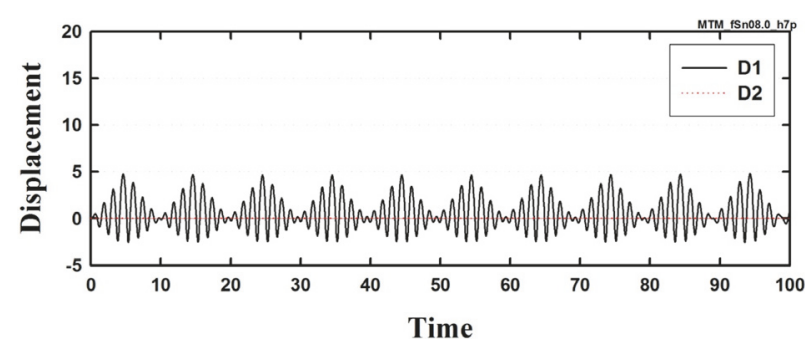

(a)

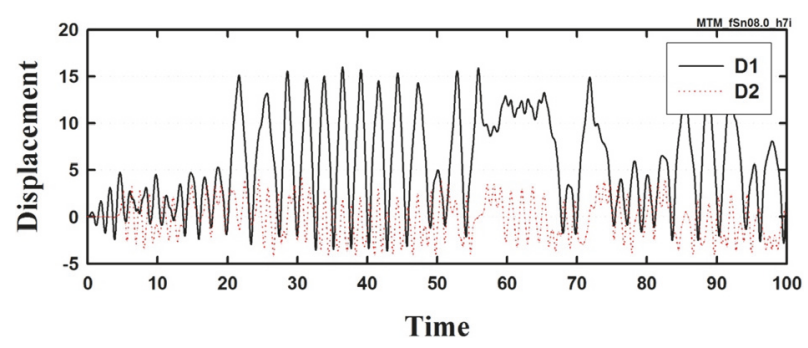

(b)

Figure 16: Transient response of shallow arches with initial imperfection under sinusoidal excitation $\left(h=7, \Lambda_{0}=8.0\right)$ : (a) perfection, $D_{2}=0.0$ and (b) initial imperfection, $D_{2}=0.001 h$.

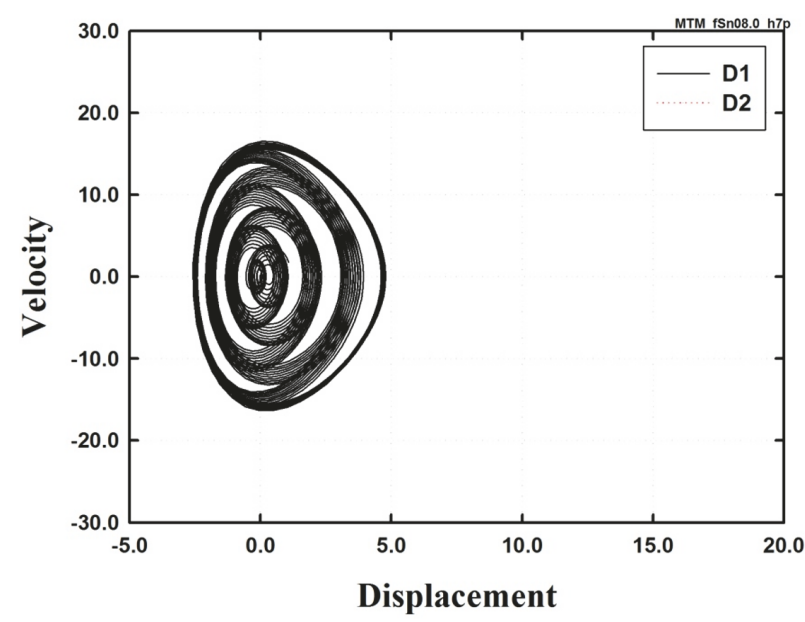

(a)

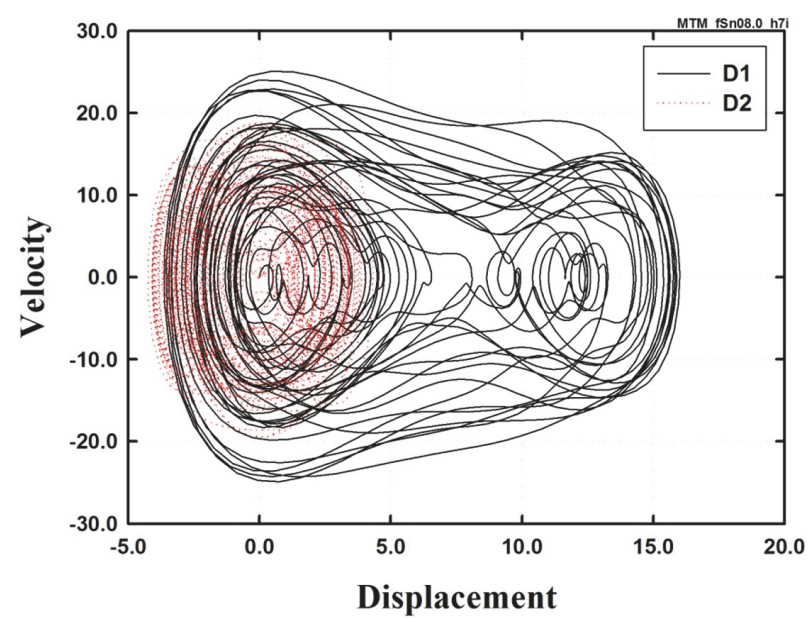

(b)

FIGURE 17: Phase diagram of the dynamic response of the analytical model with initial imperfection $\left(h=7, \Lambda_{0}=8.0\right)$ : (a) trajectory of $D_{2}=0.0$ and (b) trajectory of $D_{2}=0.001 h$.

to 15 in Figure 18(a), the amplitude of symmetric mode $D_{1}$ is increased by the perturbation of the asymmetric mode $D_{2}$. The result of transient response (Figure 18(a)) manifests the effect of coupling under asymmetric mode very clearly. Figure 18(b) shows that the attractor exhibits a very similar change to the case of sinusoidal excitation.

Analyses of dynamic response to step, sinusoidal, and beating excitation under indirect snapping condition and the influence of asymmetric mode were carried out in order to evaluate the effectiveness of the multistep Taylor series method. Coupling under nonlinearity and symmetric and asymmetric modes was well manifested in dynamic response to these excitations. The dynamic response under periodic excitation at the same period as the natural frequency $w_{0}$ was very reliable and verified.

\section{Conclusion}

This paper examined a dynamic analysis of nonlinear governing equations for such structures as shallow arches and the computational method for their analytical solution. The Taylor method was used to obtain a semianalytical solution to a motion equation, and a solution composed of a polynomial function with respect to time was formulated in multistep. A dynamic analysis was conducted to evaluate the computed solution for the behavior of shallow arches subjected to step and periodic excitations. Besides, to verify the results using the multistep Taylor series method, the 4th-order Runge-Kutta method was used to compute the responses and trajectories of the shallow arches under the excitation, and the results are well matched. The analysis result for the arch under symmetric mode revealed that the dynamic buckling load level became higher with the higher shape parameter value of the arch, and the phase diagram showed a change of attraction at the load level of direct snapping compared to the level prior to the occurrence of direct snapping. The stability of the symmetric mode arches is also summarized, and the equilibria according to $h$ are investigated. For $h \leq 2$, dynamic snapping does not happen, and the arches system for $h>4$ are very sensitive. In particular, for $h>4$, it can be clearly seen that the attractors are divided into positive and negative values. For the case of indirect buckling, the similar attractor and response, which manifested the coupling phenomenon under symmetric and asymmetric modes, were also obtained. A response result sensitive to the initial imperfection was observed in the tests of the sinusoidal and beating excitations, 


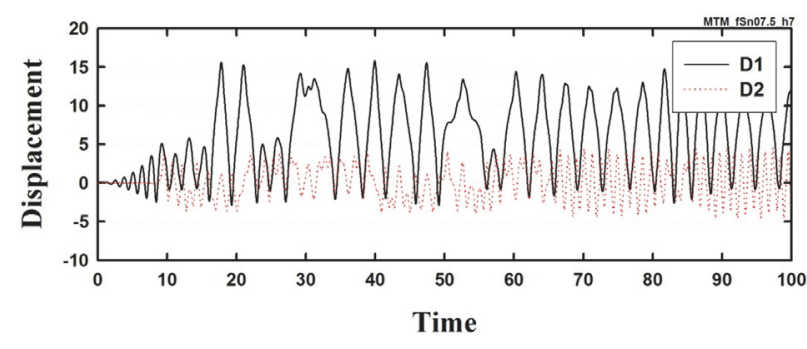

(a)

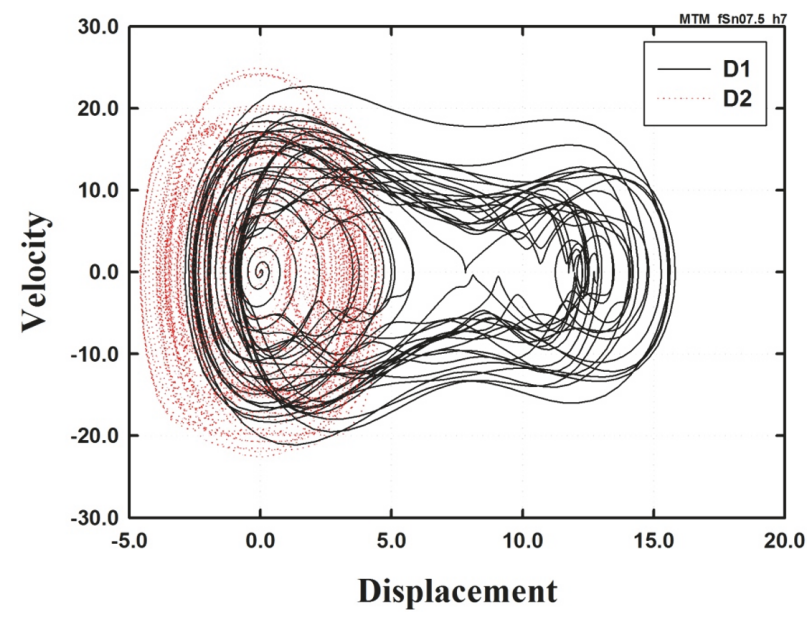

(b)

FIGURE 18: Dynamic response of shallow arches under beating excitation $\left(h=7, \Lambda_{0}=8.0\right)$ : (a) transient response of motion and (b) trajectory; initial imperfection, $D_{2}=0.001 h$.

and a change in the trajectory in the phase plan was also observed. The multistep Taylor series solution as a result of this study was valid in explaining the dynamic behavior of the direct and indirect snapping of shallow arches based on the observation of the time history response and phase plan in this study.

\section{Notations}

$y_{0}(x): \quad$ Initial shape of the arch

$y^{*}(x, t)$ : Deflection from the initial configuration

$y(x, t)$ : Vertical deflections

$u(x, t):$ Longitudinal deflection

$p(x, t)$ : External load

$(x, y): \quad$ Coordinate system of the arch

$L: \quad$ Distance of the two ends of the arch from each other

$\rho: \quad$ Density of mass

$E: \quad$ Young's modulus

$A(x): \quad$ Area of the arch's section

$I(x)$ : Moment of inertia

$T: \quad$ Kinetic energy of the arch

$U: \quad$ Potential (strain) energy of the arch $\left(=U_{a}+U_{b}\right)$

$U_{a}$ : Potential energy due to the axial force

$U_{b}$ : $\quad$ Potential energy due to bending

$\varepsilon_{0}: \quad$ Axial strain on the natural axis

$\kappa: \quad$ Curvature

$N: \quad$ Internal normal force $\left(N=E A \varepsilon_{0}\right)$

$M: \quad$ Bending moment $(M=E I \kappa)$

$W_{n c}: \quad$ Work by nonconservative system

$W_{d}: \quad$ Work by dissipative force

$W_{\text {ext }}: \quad$ Work by external force $p(x, t)$

$\xi: \quad$ Dimensionless parameter $(=\pi x / L)$

$k: \quad$ Radius of gyration of the cross-section

$\eta_{0}: \quad$ Dimensionless initial shape configuration $\left(=y_{0} / k\right)$ $\eta$ : $\quad$ Dimensionless deflection configuration $(=y / k)$

$D_{n}$ : $\quad$ Coefficient of the dimensionless deflection function

$h$ : Coefficient of the dimensionless initial shape function

$\Lambda: \quad$ Coefficient of the dimensionless excitation function

$\gamma: \quad$ Damping coefficient of the arch

$u$ : $\quad$ Dimensionless displacement of the symmetric mode $\left(=D_{1}-h\right)$

$v$ : Dimensionless velocity of the symmetric mode $(=\dot{u})$

$\delta_{i j}: \quad$ Kronecker delta

$t_{h}$ : Time step-size

$\tau$ : $\quad$ Time parameter

$\boldsymbol{R}\left(n, t, t_{0}\right)$ : Remainder term of Taylor series

$\widehat{u}$ : $\quad$ Equilibrium points of the first-order arch system

$u_{0}$ : Local minimum (or maximum) points of the

$\alpha, \beta: \quad$ Periodic parameter of the excitation function

$w_{0}$ : Natural angular frequency.

\section{Additional Points}

Highlight. Application of higher order multistep Taylor method to obtain a semianalytical solution for shallow arches. Investigation of dynamic snapping for the verification of the applicability of the multistep Taylor method. Investigation of the sensitivity of shallow arches under step, sinusoidal, and beating excitation.

\section{Conflicts of Interest}

The authors declare that they have no conflicts of interest. 


\section{Acknowledgments}

This research was supported not only by Basic Science Research Program through the National Research Foundation of Korea (NRF) funded by the Ministry of Science, ICT \& Future Planning (NRF-2016R1D1A1B03931154 and NRF2017R1D1A1B03031451) but also by Architecture \& Urban Development Research Program funded by Ministry of Land, Infrastructure and Transport of Korean government (Grant 18AUDP-B100343-04).

\section{References}

[1] N. J. Hoff and V. G. Bruce, "Dynamic analysis of the buckling of laterally loaded flat arches," Journal of Mathematical Physics, vol. 32, pp. 276-288, 1954.

[2] J. S. Humphreys, "On dynamic snap buckling of shallow arches," AIAA Journal, vol. 4, no. 5, pp. 878-886, 1966.

[3] M. H. Lock, "Snapping of a shallow sinusoidal arch under a step pressure load," AIAA Journal, vol. 4, no. 7, pp. 1249-1256, 1966.

[4] C. S. Hsu, "Equilibrium configurations of a shallow arch of arbitrary shape and their dynamic stability character," International Journal of Non-Linear Mechanics, vol. 3, no. 2, pp. 113-136, 1968.

[5] S. T. Ariaratnam and T. S. Sankar, "Dynamic snap-through of shallow arches under stochastic loads," AIAA Journal, vol. 6, no. 5, pp. 798-802, 1968.

[6] V. Sundararajan and D. S. Kumani, "Dynamic snap-buckling of shallow arches under inclined loads," AIAA Journal, vol. 10, no. 8, pp. 1090-1091, 1972.

[7] M. T. Donaldson and R. H. Plaut, "Dynamic stability boundaries for a sinusoidal shallow arch under pulse loads," AIAA Journal, vol. 21, no. 3, pp. 469-471, 1983.

[8] A. N. Kounadis, J. Raftoyiannnis, and J. Mallis, "Dynamic buckling of an arch model under impact loading," Journal of Sound and Vibration, vol. 134, no. 2, pp. 193-202, 1989.

[9] K. B. Blair, C. M. Krousgrill, and T. N. Farris, "Non-linear dynamic response of shallow arches to harmonic forcing," Journal of Sound and Vibration, vol. 194, no. 3, pp. 353-367, 1996.

[10] J. Levitas, J. Singer, and T. Weller, "Global dynamic stability of a shallow arch by Poincaré-like simple cell mapping," International Journal of Non-Linear Mechanics, vol. 32, no. 2, pp. 411424, 1997.

[11] Y.-P. Tseng, C. S. Huang, and C.-J. Lin, "Dynamic stiffness analysis for in-plane vibrations of arches with variable curvature," Journal of Sound and Vibration, vol. 207, no. 1, pp. 15-31, 1997.

[12] M. A. De Rosa and C. Franciosi, "Exact and approximate dynamic analysis of circular arches using DQM," International Journal of Solids and Structures, vol. 37, no. 8, pp. 1103-1117, 1999.

[13] Q. Bi and H. H. Dai, "Analysis of non-linear dynamics and bifurcations of a shallow arch subjected to periodic excitation with internal resonance," Journal of Sound and Vibration, vol. 233, no. 4, pp. 557-571, 2000.

[14] W. Lacarbonara and G. Rega, "Resonant non-linear normal modes. II. Activation/orthogonality conditions for shallow structural systems," International Journal of Non-Linear Mechanics, vol. 38, no. 6, pp. 873-887, 2003.

[15] X. Kong, B. Wang, and J. Hu, "Dynamic snap buckling of an elastoplastic shallow arch with elastically supported and clamped ends," Computers \& Structures, vol. 55, no. 1, pp. 163166, 1995.
[16] J.-S. Chen and J.-S. Lin, "Stability of a shallow arch with one end moving at constant speed," International Journal of Non-Linear Mechanics, vol. 41, no. 5, pp. 706-715, 2006.

[17] J.-S. Chen and Y.-T. Li, "Effects of elastic foundation on the snapthrough buckling of a shallow arch under a moving point load," International Journal of Solids and Structures, vol. 43, no. 14-15, pp. 4220-4237, 2006.

[18] J. Ha, S. Gutman, S. Shon, and S. Lee, "Stability of shallow arches under constant load," International Journal of Non-Linear Mechanics, vol. 58, pp. 120-127, 2014.

[19] L. N. Virgin, R. Wiebe, S. M. Spottswood, and T. G. Eason, "Sensitivity in the structural behavior of shallow arches," International Journal of Non-Linear Mechanics, vol. 58, pp. 212-221, 2014.

[20] H. Farokhi and M. H. Ghayesh, "Thermo-mechanical dynamics of perfect and imperfect Timoshenko microbeams," International Journal of Engineering Science, vol. 91, pp. 12-33, 2015.

[21] J.-S. Lin and J.-S. Chen, "Dynamic snap-through of a laterally loaded arch under prescribed end motion," International Journal of Solids and Structures, vol. 40, no. 18, pp. 4769-4787, 2003.

[22] B. Budiansky and R. S. Roth, "Axisymmetric dynamic buckling of clamped shallow spherical shells; collected papers on instability of shells structures," NASA TN D-1510, pp. 597-606, 1962.

[23] T. Belytschko, "A survey of numerical methods and computer programs for dynamic structural analysis," Nuclear Engineering and Design, vol. 37, no. 1, pp. 23-34, 1976.

[24] J. Argyris, M. Papadrakakis, and Z. S. Mouroutis, "Nonlinear dynamic analysis of shells with the triangular element TRIC," Computer Methods Applied Mechanics and Engineering, vol. 192, no. 26-27, pp. 3005-3038, 2003.

[25] A. Sadighi, D. D. Ganji, and B. Ganjavi, "Traveling wave solutions of the sine-gordon and the coupled sine-gordon equations using the homotopy-perturbation method," Scientia Iranica Transaction B: Mechanical Engineering, vol. 16, no. 2, pp. 189-195, 2009.

[26] R. Barrio, "Performance of the Taylor series method for ODEs/DAEs," Applied Mathematics and Computation, vol. 163, no. 2, pp. 525-545, 2005.

[27] R. Barrio, F. Blesa, and M. Lara, "VSVO formulation of the Taylor method for the numerical solution of ODEs," Computers \& Mathematics with Applications, vol. 50, no. 1-2, pp. 93-111, 2005.

[28] G. Adomian and R. Rach, "Generalization of Adomian polynomials to functions of several variables," Computers \& Mathematics with Applications, vol. 24, no. 5-6, pp. 11-24, 1992.

[29] G. Adomian and R. Rach, "Modified Adomian polynomials," Mathematical and Computer Modelling, vol. 24, no. 11, pp. 3946, 1996.

[30] S. J. Liao, The proposed Homotopy Analysis Technique for the Solution of Nonlinear Problems [Ph.D. thesis], Shanghai Jiao Tong University, 1992.

[31] J.-H. He, "Homotopy perturbation method: a new nonlinear analytical technique," Applied Mathematics and Computation, vol. 135, no. 1, pp. 73-79, 2003.

[32] J. He, "Application of homotopy perturbation method to nonlinear wave equations," Chaos, Solitons \& Fractals, vol. 26, pp. 695-700, 2005.

[33] M. S. H. Chowdhury, I. Hashim, and S. Momani, “The multistage homotopy-perturbation method: a powerful scheme for handling the Lorenz system," Chaos, Solitons \& Fractals, vol. 40, no. 4, pp. 1929-1937, 2009. 
[34] R. Barrio, M. Rodríguez, A. Abad, and F. Blesa, "Breaking the limits: the Taylor series method," Applied Mathematics and Computation, vol. 217, no. 20, pp. 7940-7954, 2011.

[35] S. Shon, S. Lee, J. Ha, and C. Cho, "Semi-analytic solution and stability of a space truss using a high-order Taylor series method," Materials, vol. 8, no. 5, pp. 2400-2414, 2015. 


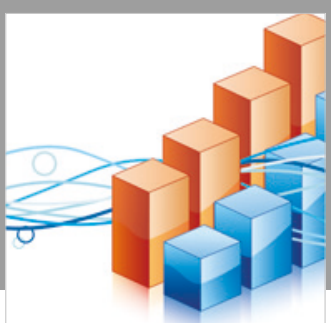

Advances in

Operations Research

\section{-n-m}
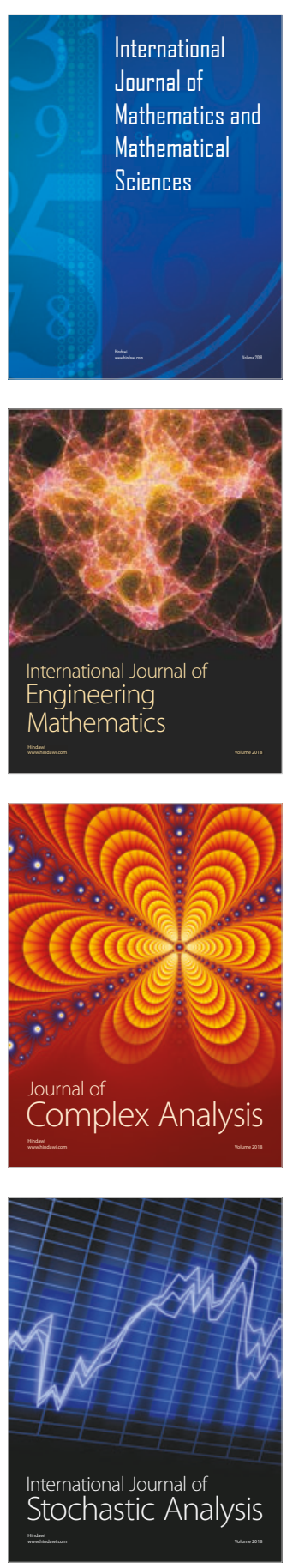
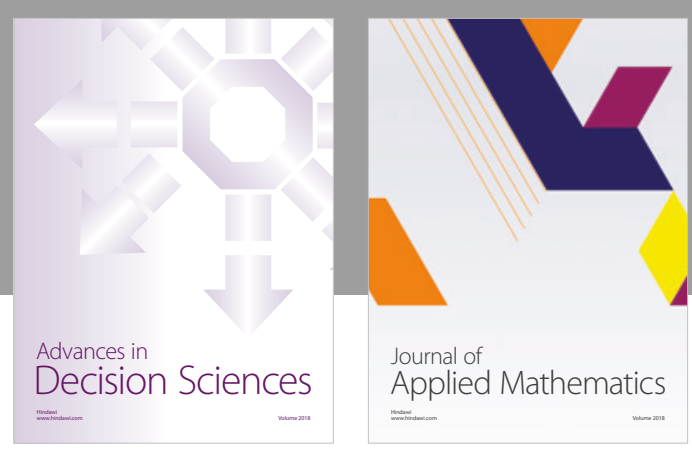

Journal of

Applied Mathematics
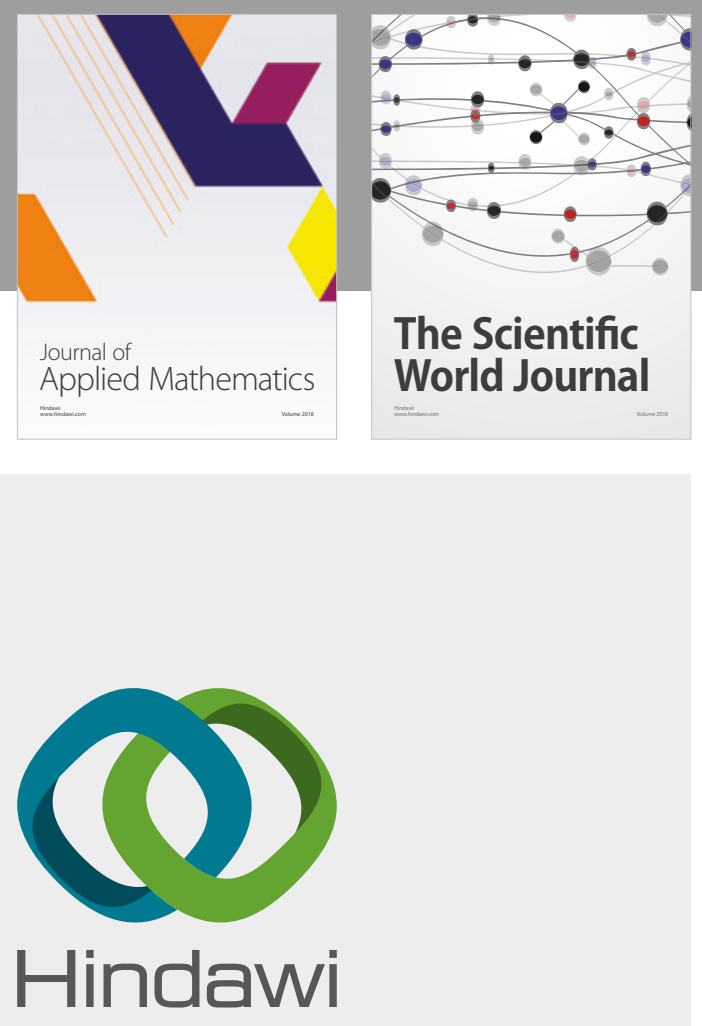

Submit your manuscripts at

www.hindawi.com

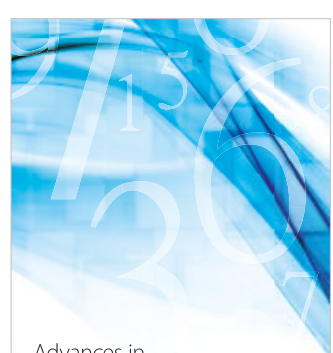

Advances in
Numerical Analysis
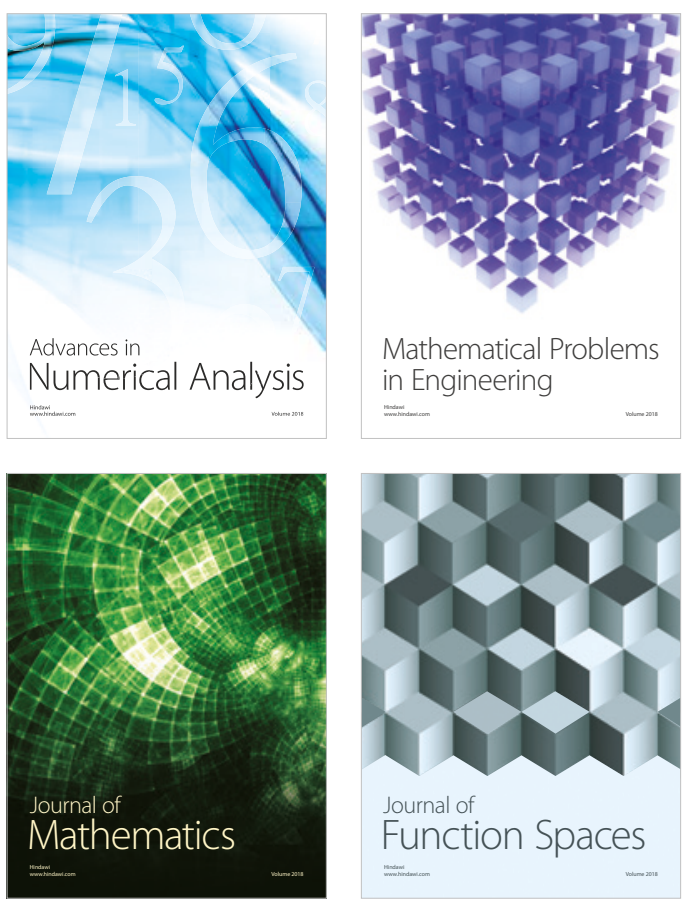

Mathematical Problems in Engineering

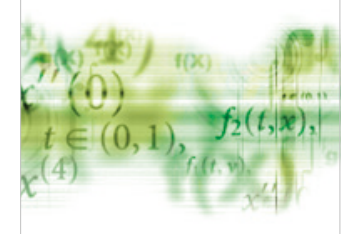

International Journal of

Differential Equations

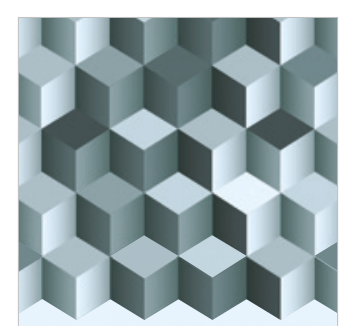

Journal of

Function Spaces

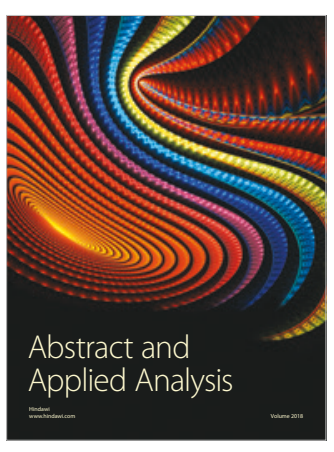

The Scientific

World Journal

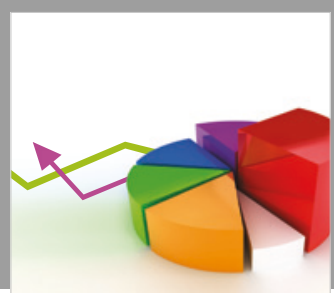

Journal of

Probability and Statistics
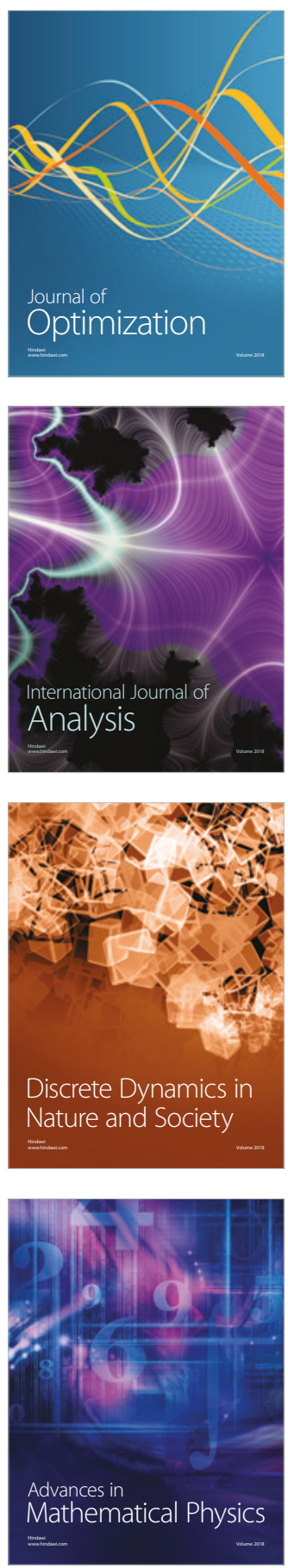